QUARTERLY OF APPLIED MATHEMATICS

VOLUME LXVII, NUMBER 2

JUNE 2009, PAGES 221-247

S 0033-569X(09)01101-0

Article electronically published on March 27, 2009

\title{
RECOVERY OF MULTIPLE OBSTACLES BY PROBE METHOD
}

\author{
BY
}

JIN CHENG (School of Mathematical Sciences, Fudan University, Shanghai 200433, People's Republic of China),

JIJUN LIU (Department of Mathematics, Southeast University, Nanjing 210096, People's Republic of China),

GEN NAKAMURA (Department of Mathematics, Graduate School of Science, Hokkaido University, Sapporo 060-0810, Japan),

AND

SHENGZHANG WANG (Department of Mechanics and Engineering Science, Fudan University, Shanghai 200433, People's Republic of China)

Abstract. We consider an inverse scattering problem for multiple obstacles $D=$ $\bigcup_{j=1}^{N} D_{j} \subset R^{3}$ with different types of boundary for $D_{j}$. By constructing an indicator function from the far-field pattern of the scattered wave, we can firstly reconstruct the shape of all obstacles, then identify the type of boundary for each obstacle, as well as the boundary impedance in the case that obstacles have the Robin-type boundary condition. The novelty of our probe method compared with the existing probe method is that we succeeded in identifying the type of boundary condition for multiple obstacles by analyzing the behavior of both the imaginary part and the real part of the indicator function. The numerical realizations are given to show the performance of this inversion method.

1. Introduction. Let $D$ be a bounded domain in $R^{3}$ such that $D=\bigcup_{j=1}^{N} D_{j}, \overline{D_{i}} \cap$ $\overline{D_{j}}=\emptyset(i \neq j)$. Each $D_{j}$ is a simply connected domain with $C^{2}$ boundary $\partial D_{j}$. The scattering of time-harmonic acoustic plane waves by the obstacle $D$ with some boundary

Received September 7, 2007.

2000 Mathematics Subject Classification. Primary 35R30, 35J05, 76Q05.

Key words and phrases. Inverse scattering, probe method, uniqueness, indicator, numerics.

The first author is partly supported by NSFC (No.9207011148, No.3007011043).

The second author is supported by NSFC (No.10371018).

The third author is partly supported by Grant-in-Aid for scientific research, Ministry of Education, Science and Culture, Japan (No.12640153).

The fourth author is the corresponding author for this paper.

E-mail address: jcheng@fudan.edu.cn

E-mail address: jjliu@seu.edu.cn

E-mail address: gnaka@math.sci.hokudai.ac.jp

E-mail address: szwang@fudan.edu.cn 
is modelled as an exterior boundary value problem for the Helmholtz equation. That is, for a given incident plane wave $u^{i}(x)=e^{i k x \cdot d}, d \in S^{2}=\left\{\xi \in R^{3}:|\xi|=1\right\}$, the total wave field $u=u^{i}+u^{s} \in H_{l o c}^{1}\left(R^{3} \backslash \bar{D}\right)$ satisfies

$$
\begin{cases}\Delta u+k^{2} u=0, & \text { in } R^{3} \backslash \bar{D}, \\ B u(x, t)=0, & \text { on } \partial D, \\ \frac{\partial u^{s}}{\partial r}-i k u^{s}=O\left(\frac{1}{r}\right), & r=|x| \longrightarrow \infty,\end{cases}
$$

where $B$ is a boundary operator corresponding to different types of the obstacles $D_{j}(1 \leq$ $j \leq N)$, that is,

$$
B u= \begin{cases}u & \text { if } \partial D_{j} \text { is sound-soft } \\ \frac{\partial u}{\partial \nu} & \text { if } \partial D_{j} \text { is sound-hard, } \\ \frac{\partial u}{\partial \nu}+i \sigma(x) u & \text { if } \partial D_{j} \text { is Robin-type }\end{cases}
$$

where $\nu$ is the unit normal on $\partial D_{j}$ directed into the exterior of $D_{j}$, and $\sigma(x)>0$ is the boundary impedance coefficient. By the results in [5], we know that there exists a unique solution for the forward scattering problem (1.1).

For the incident field $u^{i}(x)=e^{i k x \cdot d}$, the far-field pattern $u^{\infty}(\theta, d)$ can be defined by

$$
u^{s}(x)=\frac{e^{i k|x|}}{|x|}\left\{u^{\infty}(\theta, d)+O\left(\frac{1}{|x|}\right)\right\}, \quad|x| \longrightarrow \infty,
$$

where $\theta, d \in S^{2}$.

Generally, the inverse scattering problem corresponding to (1.1) is to identify the boundary $\partial D$ and also $\sigma(x)$ in the case of a Robin-type boundary, from a knowledge of the far-field pattern. If $D$ is just one obstacle, then identifying $\partial D$ for each kind of boundary conditions has been discussed thoroughly. For example, if $D$ is soundsoft (Dirichlet boundary condition on $\partial D$ ) or sound-hard (Neumann boundary condition on $\partial D$ ), the problems have been studied by many researchers; see [3, 6], 10, [1], [13, [18, 22]. In the case of an obstacle with Robin-type boundary, the problem of reconstructing $\sigma(x)$, when $\partial D$ is given, has also been studied; see [4, 6], 20], 21]. For the inverse scattering problem of determining both $\partial D$ and the boundary impedance, an approximate determination (or reconstruction) of the shape of $D$ and the boundary impedance was discussed in 24] by using the asymptotic behavior of the low frequency scattered waves associated with three different incident waves (or frequencies). In [16], one numerical method is proposed to determine both $\partial D$ and the impedance $\sigma(x)$. In [1, the authors gave a uniqueness and reconstruction formula for identifying $\partial D$ and the impedance for a Robin-type obstacle from the far-field pattern, by applying the probe method introduced by M. Ikehata (see [10, 11, [12, 13] and 14 for example). Moreover, it has also been noticed that the probe method, the singular sources method given in 23, as well as the factorization method 15, can be applied to determine the boundaries of multiple obstacles if their boundary types are the same (sound-soft or sound-hard). Now, we consider another problem: if there are many obstacles with different types of boundary such as sound-soft, sound-hard, as well as Robin-type, can we still identify their shapes and locations as well as the type of boundary for each obstacle? What is the numerical performance of the inversion scheme in this multiple-obstacle case? In [17] and [9, the theory of the factorization method was adapted and applied to 
identify the shapes and locations of obstacles. Its numerical examples were given in [17. However, the types of boundaries were not identified. So, the aim of this paper is to give a reconstruction formula for identifying not only the shapes and locations of obstacles, but also their boundary types at the same time.

Our result can be stated as follows.

Theorem 1.1. Let $D$ be a bounded domain consisting of finite obstacles $D_{j}(j=$ $1,2, \ldots, N)$, namely, $D=\bigcup_{j=1}^{N} D_{j}$. We assume that each obstacle $D_{j}$ is a simply connected bounded domain with $C^{2}$ boundary $\partial D_{j}$ and $\bar{D}_{i} \cap \bar{D}_{j}=\emptyset$ for $i \neq j$. For given incident plane waves $u^{i}(x, d)=e^{i k x \cdot d}$, consider the following scattering problem for the total wave field $u(x, d)=u^{i}(x, d)+u^{s}(x, d)$ :

$$
\begin{cases}\Delta u+k^{2} u=0, & \text { in } R^{3} \backslash \bar{D}, \\ B_{j} u(x, t)=0, & \text { on } \partial D_{j}, j=1,2, \ldots, N, \\ \frac{\partial u^{s}}{\partial r}-i k u^{s}=O\left(\frac{1}{r}\right), & r=|x| \longrightarrow \infty,\end{cases}
$$

where $B_{j}$ is one of the boundary operators in (1.2) for $j=1,2, \ldots, N$. Assume that $\bar{D} \subset \Omega$ for some known sphere $\Omega$ and $0<\sigma_{j}(x) \in C^{1}\left(\partial D_{j}\right)$ for Robin-type obstacles $D_{j}$. If there exists at least one Robin-type obstacle, then from the far-field pattern $u^{\infty}(\theta, d)$ for all $\theta, d \in S^{2}$, we can

(1) determine the number of obstacles $N$,

(2) reconstruct $\partial D_{j}$ for $j=1,2, \ldots, N$,

(3) identify the type of each obstacle $D_{j}$,

(4) reconstruct $\sigma_{j}(x)$ for the Robin-type obstacles $D_{j}$.

A similar result holds for the two dimensional case. The numerical performance of this method is given for the two dimensional case in the last section. Our main tool to deal with this problem is still the probe method. This method gives a reconstruction formula for the shape and location of an obstacle by using the indicator function and analyzing its behavior. However, there are some new ingredients in this paper. In the case of multiple obstacles, we not only have to determine the shape and location of each obstacle, but also we have to determine the number of obstacles and identify the type of each obstacle. This is the major and important difference between the multiple-obstacle inversion and the single-obstacle one. Especially, we have to extract some characteristics of the indicator function such that we can distinguish sound-hard obstacles and obstacles with Robin-type boundary, since in most cases, we can consider the Neumann boundary as a special case of the Robin boundary with $\sigma(x)=0$. Then the most important ingredient of this paper is that we succeeded in providing a method distinguishing the sound-hard boundary and the Robin-type boundary. More precisely, we can determine the positions of obstacles and identify the sound-soft boundary from the real part of the indicator function, while distinguishing the sound-hard boundary from the Robintype boundary is done by considering the imaginary part of the indicator function. In order to carry this out, the most important and difficult thing is to rewrite the indicator function in an appropriate form and analyze its behavior. The number of obstacles can be obtained immediately when we get the whole image of all obstacles. 
We will give a mathematically rigorous reconstruction formula for recovering $\partial D_{j}$ for $j=1,2, \ldots, N$ and their boundary types. Then the uniqueness of identifying $\partial D_{j}$ and the determination of the number of obstacles from $u^{\infty}(d, \theta)$ for all $d, \theta \in S^{2}$ becomes obvious from the reconstruction. Since our reconstruction procedure is pointwise, it is enough to consider the case that $D$ consists of 3 obstacles with sound-soft, sound-hard and Robin-type boundary, respectively, and to illustrate the reconstruction procedure for identifying the location and type for each obstacle. This does not lose any generality. More precisely, we assume that $D_{1}, D_{2}, D_{3}$ are sound-soft, sound-hard and Robin-type, respectively. Once we have identified the shape, location and type of each obstacle, we determine $\sigma(x):=\sigma_{3}(x)$ on $\partial D_{3}$ by the moment method. So, henceforth we assume $N=3$.

The numerical performance of our method for the two dimensional case was tested for 3 circle shaped obstacles with different types of boundaries using the numerical realization scheme of the probe method given in [8. The numerical result shows that we can reconstruct a little more than a convex hull of multiple obstacles only; the neighboring parts of different obstacles are hard to recover. However, the center of the obstacles and the boundary types of the obstacles can be identified.

REMARK 1.2. For our problem, if $\sigma(x) \in C^{1}\left(\partial D_{3}\right)$, the well-posedness for this direct problem can be established from the standard scattering theory. That is, we can apply the radiation condition to get the uniqueness (Theorem 3.12, 5. and use the combined single-layer and double-layer theory to get the existence of the solution.

Remark 1.3. We can also identify $D_{1}$ and $D_{2}$ in case of $D_{3}=\emptyset$ if we assume the unique solvability of the boundary value problem (2.2) given later.

REMARK 1.4. Our reconstruction method considers the inverse scattering problem with multiple obstacles with different types of boundary by firstly transforming the farfield pattern of the scattered wave to the Dirichlet-to-Neumann (D-to-N) map defined on the surface of a ball, and then recovering the obstacles from the D-to- $\mathrm{N}$ map by the probe method. The main difficulties are that we have to solve an integral equation of the second kind with hyper-singular kernel (see (2.4) in the sequel) from the far-field pattern to construct the D-to-N map and to provide a numerically feasible method for the Runge approximation. The first problem can be solved by the technique proposed in [19], and the second one has been solved recently by the optimization technique ([2]).

Our paper is organized as follows:

- Section 2: Preliminary results

- Section 3: Probe method

- Section 4: Moment method for determining $\sigma(x)$

- Section 5: Some estimates

- Section 6: Singularity analysis

- Section 7: Numerical performance

2. Preliminary results. In this section, we give some known results for the probe method, which are necessary for our paper. 
Without loss of generality, we assume that $\bar{D} \subset B\left(0, \frac{R}{2}\right)$ for some constant $R>0$. We also assume that 0 is not a Dirichlet eigenvalue of $\Delta+k^{2}$ in $\Omega:=B(0, R)$ for given $k>0$.

Proposition 2.1. The scattered solution $u^{s}(x, d)$ for $|x|>\frac{R}{2}$ can be determined uniquely from $u^{\infty}(d, \theta)$.

The physical background for this proposition is obvious; that is, the far-field pattern of the scattered wave determines the near-field pattern outside the obstacle completely. This procedure has nothing to do with the boundary conditions of scatterers. For the proof, see Theorem 3.6 and Corollary 3.8 in [5] or [1].

Let $G(x, y)=\frac{e^{i k|x-y|}}{4 \pi|x-y|}$ be the fundamental solution of the Helmholtz equation. For each $y \in R^{3} \backslash \bar{D}$, we define $E(\cdot, y) \in H_{l o c}^{1}\left(R^{3} \backslash \bar{D}\right)$ as the solution to

$$
\begin{cases}\Delta E+k^{2} E=0, & \text { in } R^{3} \backslash \bar{D}, \\ B_{i} E(x, y)=-B_{i} G(x, y), & \text { on } \partial D_{i}, \quad i=1,2,3, \\ \frac{\partial E}{\partial r}-i k E=O\left(\frac{1}{r}\right), & r=|x| \longrightarrow \infty .\end{cases}
$$

Proposition 2.2. For $x, y \in \partial \Omega, E(x, y), \frac{\partial}{\partial \nu(x)} E(x, y)$ and $\frac{\partial}{\partial \nu(y)} E(x, y)$ can be determined from $u^{\infty}(d, \theta)$ for all $d, \theta \in S^{2}$.

The proof for $D=D_{1} \cup D_{2} \cup D_{3}$ given here is analogous to that given in [1] for $D=D_{3}$.

Proof. In fact, since we can choose $R$, so we assume that 0 is not the Dirichlet eigenvalue of $\Delta+k^{2}$. Therefore $\left\{e^{i k x . d} \mid d \in S^{2}\right\}$ is dense in $L^{2}(\partial \Omega)$ ([6], Theorem 5.5). For any fixed $y \in \partial B\left(0, R_{1}\right)\left(R_{1}>R\right)$, there exists a sequence $\left\{\alpha_{j}^{n}(y), d_{j}^{n}(y)\right\}$ such that

$$
\sum_{1 \leq j \leq m_{n}(y)} \alpha_{j}^{n}(y) e^{i k x \cdot d_{j}^{n}(y)} \rightarrow G(x-y) \quad \text { in } \quad L^{2}(\partial \Omega)
$$

as $n \rightarrow \infty$. On the other hand, since both $\sum_{1 \leq j \leq m_{n}(y)} \alpha_{j}^{n}(y) e^{i k x \cdot d_{j}^{n}(y)}$ and $G(x-y)$ satisfy the Helmholtz equation in $\Omega$, by the result in [6] (Theorem 5.4), we know that $\sum_{1 \leq j \leq m_{n}(y)} \alpha_{j}^{n}(y) e^{i k x \cdot d_{j}^{n}(y)} \rightarrow G(x-y)$ uniformly on any compact subset of $\Omega$ (together with all their derivatives). Therefore it follows that

$$
B_{i} \sum_{1 \leq j \leq m_{n}(y)} \alpha_{j}^{n}(y) e^{i k x \cdot d_{j}^{n}(y)} \rightarrow B_{i} G(x-y)
$$

in $L^{2}\left(\partial D_{i}\right), i=1,2,3$ as $n \rightarrow \infty$. Since $u^{s}(x, d) \in H^{1}\left(R^{3} \backslash \bar{D}\right)$ satisfies

$$
\begin{cases}\Delta u^{s}+k^{2} u^{s}=0, & \text { in } \quad R^{3} \backslash \bar{D}, \\ B_{i} u^{s}=-B_{i} e^{i k x \cdot d}, & \text { on } \partial D_{i}, \quad i=1,2,3, \\ \frac{\partial u^{s}}{\partial r}-i k u^{s}=O\left(\frac{1}{r}\right), & r=|x| \rightarrow \infty\end{cases}
$$

by the continuous dependence of the direct scattering problem, we have

$$
\sum_{1 \leq j \leq m_{n}(y)} \alpha_{j}^{n}(y) u^{s}\left(x, d_{j}^{n}(y)\right) \rightarrow E(x, y) \text { uniformly on } R / 2<|x|<2 R
$$


for $y \in \partial B\left(0, R_{1}\right)$. Now we get from Proposition 1 that, for $y \in \partial B\left(0, R_{1}\right)$ and $R / 2<$ $|x|<2 R, E(x, y), \frac{\partial E(x, y)}{\partial \nu(x)}, \frac{\partial E(x, y)}{\partial \nu(y)}$ can be determined by $\left\{u^{\infty}(d, \theta) \mid \theta, d \in S^{2}\right\}$. Since $R_{1}$ is arbitrary, we complete the proof by letting $R_{1} \rightarrow R$.

Consider a solution $u(x) \in H^{1}(\Omega \backslash \bar{D})$ to the following boundary value problem:

$$
\begin{cases}\Delta u+k^{2} u=0, & \text { in } \Omega \backslash \bar{D}, \\ B_{j} u(x, t)=0, & \text { on } \partial D_{j}, j=1,2,3, \\ u(x)=f, & \text { on } \partial \Omega\end{cases}
$$

for given $f \in H^{1 / 2}(\partial \Omega)$.

Since we have used $\bar{D}$ to indicate the closure of the domain $D$, we will use $\tilde{z}$ to indicate the complex conjugate of the complex number $z$ in the sequel.

Lemma 2.3. If $D_{3} \neq \emptyset$, then there exists a unique solution to (2.2) for any $f \in H^{1 / 2}(\partial \Omega)$.

Proof. Firstly, we prove the uniqueness. It is enough to prove that $f=0$ implies $u=0$ in $\Omega \backslash \bar{D}$. For $f=0$, it is easy to see from (2.2) that

$$
\begin{aligned}
& 0=\int_{\Omega \backslash \bar{D}}\left(\Delta u+k^{2} u\right) \tilde{u} d x=\int_{\partial D} \tilde{u} \frac{\partial u}{\partial \nu} d s-\int_{\Omega \backslash \bar{D}}\left(\nabla u \cdot \nabla \tilde{u}-k^{2} u \tilde{u}\right) d x, \\
& 0=\int_{\Omega \backslash \bar{D}}\left(\Delta \tilde{u}+k^{2} \tilde{u}\right) u d x=\int_{\partial D} u \frac{\partial \tilde{u}}{\partial \nu} d s-\int_{\Omega \backslash \bar{D}}\left(\nabla \tilde{u} \cdot \nabla u-k^{2} \tilde{u} u\right) d x
\end{aligned}
$$

due to $u=0$ on $\partial \Omega$. Subtracting these two equalities and noticing the boundary conditions of $u$ on $\partial D_{1}$ and $\partial D_{2}$ lead to

$$
\int_{\partial D_{3}}\left(\tilde{u} \frac{\partial u}{\partial \nu}-u \frac{\partial \tilde{u}}{\partial \nu}\right) d s=0
$$

Now the boundary condition in $\partial D_{3}$ leads to

$$
\int_{\partial D_{3}} \sigma(x)|u(x)|^{2} d s=0
$$

from which we get $u=0$ on $\partial D_{3}$ and $\frac{\partial u}{\partial \nu}=0$ on $\partial D_{3}$ from the boundary condition. Now the uniqueness of the Cauchy problem for the Helmholtz equations outside $D_{3}$ implies $u=0$ in $\Omega \backslash \bar{D}$. On the other hand, by the integral equation method for the scattering problem $([5,6]$ ), we know that the direct problem (2.2) can be transformed into a Fredholm integral equation of the second kind; therefore the uniqueness implies the existence due to the Fredholm alternative theorem.

REMARK 2.4. The existence of $D_{3}$ is important to the proof of our uniqueness. If we do not have an obstacle with Robin-type boundary, i.e., $D=D_{1} \cup D_{2}$, then we have to assume the uniqueness of a solution in $H^{1}(\Omega \backslash \bar{D})$ to

$$
\begin{cases}\Delta u+k^{2} u=0 & \text { in } \Omega \backslash\left(D_{1} \cup D_{2}\right), \\ B_{i} u=0 & \text { on } \partial D_{i}, i=1,2, \\ u(x)=f & \text { on } \partial \Omega .\end{cases}
$$

Define the Dirichlet-to-Neumann map $\Lambda_{\partial D, \sigma}$ formally by

$$
\Lambda_{\partial D, \sigma}:\left.f \longrightarrow \frac{\partial u}{\partial \nu}\right|_{\partial \Omega} \in H^{-1 / 2}(\partial \Omega),
$$


where $u \in H^{1}(\Omega \backslash \bar{D})$ is the solution of (2.2) for $f \in H^{1 / 2}(\partial \Omega)$. In the next lemma, we show the relations between the far-field patterns and the Dirichlet-to-Neumann map.

Lemma 2.5. Let $u$ be the solution to (2.2) for $f \in H^{1 / 2}(\partial \Omega)$. Then, $\left.\frac{\partial u}{\partial \nu}\right|_{\partial \Omega}$ can be obtained from $f(x)$ and $u^{\infty}(d, \theta)$ for $\theta, d \in S^{2}$.

Proof. Let $x_{0} \in \partial B\left(0, R_{0}\right)$ for $R / 2<R_{0}<R$. By Green's formula, we have that, for $G_{D}=G_{D}\left(x, x_{0}\right)=G\left(x, x_{0}\right)+E\left(x, x_{0}\right)$,

$$
\begin{aligned}
u\left(x_{0}\right) & =\int_{\partial \Omega}\left(G_{D} \frac{\partial u}{\partial \nu_{1}}-u \frac{\partial G_{D}}{\partial \nu_{1}}\right) d s+\sum_{j=1}^{3} \int_{\partial D_{j}}\left(G_{D} \frac{\partial u}{\partial \nu_{1}}-u \frac{\partial G_{D}}{\partial \nu_{1}}\right) d s \\
& =\int_{\partial \Omega}\left(G_{D} \frac{\partial u}{\partial \nu_{1}}-f \frac{\partial G_{D}}{\partial \nu_{1}}\right) d s
\end{aligned}
$$

because $B_{j} G_{D}=B_{j} u=0$ on $\partial D_{j}$, where $\nu_{1}$ is the outward normal to the boundary of the domain $\Omega \backslash \bar{D}$.

Taking the normal derivatives of $u$ on $\partial B\left(0, R_{0}\right)$ in the above expression and letting $R_{0} \rightarrow R$, by the properties of the single layer potential and the double layer potential ([5]), we have

$$
\frac{1}{2} \frac{\partial u\left(x_{0}\right)}{\partial \nu_{1}\left(x_{0}\right)}=\int_{\partial \Omega} \frac{\partial G_{D}\left(x, x_{0}\right)}{\partial \nu_{1}\left(x_{0}\right)} \frac{\partial u(x)}{\partial \nu_{1}(x)} d s-\frac{\partial}{\partial \nu_{1}\left(x_{0}\right)} \int_{\partial \Omega} f(x) \frac{\partial G_{D}\left(x, x_{0}\right)}{\partial \nu_{1}(x)} d s
$$

for $x_{0} \in \partial B(0, R)=\partial \Omega$. The equation (2.4) is a Fredholm integral equation of the second kind with respect to $\left.\frac{\partial u(x)}{\partial \nu(x)}\right|_{\partial \Omega}$. There exists a unique solution due to the unique solvability of (2.2).

By Proposition 2.2, we know that, for $x, y \in \partial \Omega, \frac{\partial E(x, y)}{\partial \nu(x)}$ and $\frac{\partial E(x, y)}{\partial \nu(y)}$ can be obtained from $u^{\infty}(d, \theta), \theta, d \in S^{2}$. Therefore $\left.\frac{\partial u}{\partial \nu}\right|_{\partial \Omega}$ can be obtained from $u^{\infty}(d, \theta), \theta, d \in S^{2}$ and $f(x)$. The proof is complete.

From this lemma, we see that the original inverse problem can be restated as the problem of reconstructing the shapes of the 3 obstacles and the boundary impedance of $D_{3}$ from the Dirichlet-to-Neumann map $\Lambda_{\partial D, \sigma}$.

REMARK 2.6. The Dirichlet-to-Neumann map $\Lambda_{\partial D, \sigma}$ can be defined by the following weak form.

For any $g \in H^{1 / 2}(\partial \Omega)$, take any $v \in H^{1}(\Omega)$ with $\left.v\right|_{\partial \Omega}=g$. Then it follows that

$$
\begin{aligned}
\left\langle\Lambda_{\partial D, \sigma} f, g\right\rangle & =\int_{\Omega \backslash \bar{D}}\left(\nabla u \cdot \nabla v-k^{2} u v\right) d x+\int_{\partial D} v \frac{\partial u}{\partial \nu} d s(x) \\
& =\int_{\Omega \backslash \bar{D}}\left(\nabla u \cdot \nabla v-k^{2} u v\right) d x+\int_{\partial D_{1}} v \frac{\partial u}{\partial \nu} d s(x)-\int_{\partial D_{3}} i \sigma(x) u v d s(x)
\end{aligned}
$$

for any $f \in H^{1 / 2}(\partial \Omega)$, where $u$ is the solution to (2.2) for $f \in H^{1 / 2}(\partial \Omega)$.

Corresponding to the case $D=\emptyset$, we can formally define the Dirichlet-to-Neumann map $\Lambda_{0,0}: H^{1 / 2}(\partial \Omega) \longrightarrow H^{-1 / 2}(\partial \Omega)$ by

$$
\Lambda_{0,0}:\left.f \longrightarrow \frac{\partial u_{1}}{\partial \nu}\right|_{\partial \Omega}
$$


where $u_{1}(x) \in H^{1}(\Omega)$ is the solution to

$$
\begin{cases}\Delta u_{1}+k^{2} u_{1}=0, & \text { in } \Omega \\ u_{1}(x)=f \in H^{1 / 2}(\partial \Omega), & \text { on } \partial \Omega .\end{cases}
$$

Here note that by the assumption that 0 is not the Dirichlet eigenvalue of the operator $\Delta+k^{2}$ in $\Omega$, (2.6) is uniquely solvable.

The weak formula of $\Lambda_{0,0}$ is given by

$$
\left\langle\Lambda_{0,0} f, g\right\rangle=\int_{\Omega}\left(\nabla u_{1} \cdot \nabla v-k^{2} u_{1} v\right) d x,
$$

where $u_{1}$ is the solution of (2.6) for $f \in H^{1 / 2}(\partial \Omega)$ and $v \in H^{1}(\Omega)$ satisfies $\left.v\right|_{\partial \Omega}=g$ for $g \in H^{1}(\partial \Omega)$. For the solution $u$ of (2.2) and the solution $u_{1}$ of (2.6), we have

Lemma 2.7. Let $u \in H^{1}(\Omega \backslash \bar{D})$ and $u_{1} \in H^{1}(\Omega)$ be the solutions to (2.2) and (2.6), respectively. There exists a constant $C=C\left(k, R, \sigma_{0}\right)$ such that, for all $f \in H^{1 / 2}(\partial \Omega)$,

$$
\left\|u-u_{1}\right\|_{H^{1}(\Omega \backslash \bar{D})} \leq C\left\|u_{1}\right\|_{H^{1}(D)},
$$

where $\sigma_{0}>0$ is a constant satisfying $0<\sigma(x) \leq \sigma_{0}, x \in \partial D_{3}$.

The proof of Lemma 2.7 is almost the same as that given in [12. But for the reader's convenience we give the proof in Section 5 .

\section{Probe method.}

Definition 3.1. For any non-self-intersecting continuous curve $c=\{c(t) \mid 0 \leq t \leq 1\}$, if it satisfies $c(0), c(1) \in \partial \Omega$ and $c(t) \in \Omega \quad(0<t<1)$, then we call $c$ a needle in $\Omega$.

Definition 3.2. For any needle $c$ in $\Omega$, we call

$$
t(c, D)=\sup \{0<t<1 \mid c(s) \in \Omega \backslash \bar{D} \text { for all } 0<s<t\}
$$

the geometric impact parameter (GIP). It is obvious that $t(c, D)=1$ if $c$ does not touch any point on $\partial D$.

From this definition, we know that if a needle $c$ touches $\bar{D}$, then $t(c, D)<1$ and $t(c, D)$ is the first hitting time, i.e., $c(t(c, D)) \in \partial D$ and $c(t) \in \Omega \backslash \bar{D}$ for $0<t<t(c, D)$ if we consider $t$ as a time.

Since $\Omega \backslash \bar{D}$ is connected, we have a reconstruction algorithm for $\partial D$ in terms of the geometric impact parameter and the needle, i.e.,

$$
\partial D=\{c(t) \mid t=t(c, D), c \text { is a needle and } t(c, D)<1\} .
$$

Therefore, in order to reconstruct $\partial D$, it suffices to consider the problem of calculating the GIP for each needle from the Dirichlet-to-Neumann map.

Lemma 3.3. Suppose that $\Gamma$ is an arbitrary open set of $\partial \Omega$. For each $0<t<1$, there exists a sequence $\left\{v_{n}\right\}_{n=1}^{\infty}$ in $H^{1}(\Omega)$, which satisfies

$$
\Delta v_{n}+k^{2} v_{n}=0
$$

such that $\operatorname{supp}\left(\left.v_{n}\right|_{\partial \Omega}\right) \subset \Gamma$ and

$$
v_{n} \longrightarrow G(\cdot-c(t)) \quad \text { in } \quad H_{l o c}^{1}\left(\Omega \backslash c_{t}\right),
$$

where $c_{t}:=\left\{c\left(t^{\prime}\right) ; 0<t^{\prime} \leq t\right\}$. 
This result comes from the Runge approximation theorem; see [10, [1].

REMARK 3.4. Usually the Runge approximation is not constructive, because its proof is done by using the unique continuation and Hahn-Banach theorem. However, for the Helmholtz equation, it is possible to make the Runge approximation constructive by using the translation theory (see [7]).

It is obvious that $\left.v_{n}\right|_{\partial \Omega}$ depends on $c(t)$. We denote it by $\left.v_{n}\right|_{\partial \Omega}=f_{n}(\cdot, c(t))$, where $f_{n}(\cdot, c(t)) \in H^{1 / 2}(\partial \Omega)$ and $\operatorname{supp}\left(f_{n}(\cdot, c(t))\right) \subset \Gamma$.

Definition 3.5. For a given needle $c$ in $\Omega$ and $0<t<1$, we define the indicator function

$$
I(t, c)=\lim _{n \longrightarrow \infty} \hat{I}_{n}(t, c)
$$

with

$$
I_{n}(t, c):=\langle\overbrace{\left(\Lambda_{\partial D, \sigma}-\Lambda_{0,0}\right) f_{n}(\cdot, c(t))}, f_{n}(\cdot, c(t))\rangle
$$

whenever it is defined, where $\langle\cdot, \cdot\rangle$ is the pairing between $H^{-1 / 2}(\partial \Omega)$ and $H^{1 / 2}(\partial \Omega)$, and $\overbrace{\left(\Lambda_{\partial D, \sigma}-\Lambda_{0,0}\right) f_{n}(\cdot, c(t))}$ is the complex conjugate of $\left(\Lambda_{\partial D, \sigma}-\Lambda_{0,0}\right) f_{n}(\cdot, c(t))$.

Next we show that $\Re I(t, c)$ and $\Im I(t, c)$ ( $\Re$, denote the real part and imaginary part respectively) can be used to calculate GIP from which the shapes and locations of 3 obstacles can be determined, and we can also identify the type of each obstacle.

THEOREM 3.6. For a given needle $c(t)$ in $\Omega$, it follows that

(A) $t(c, D)=1$ if and only if $I(t, c)$ exists for all $0 \leq t \leq 1$ and

$$
\sup _{0 \leq t \leq 1}|\Re I(t, c)|<+\infty .
$$

(B) $T=t(c, D)$ can be characterized by

(1) $I(t, c)$ exists for all $0 \leq t<T$ and

$$
\sup _{0 \leq s \leq t}|\Re(I(s, c))|<+\infty, \quad \text { for } \quad 0 \leq t<T,
$$

(2) $\lim _{t \rightarrow T-}|\Re I(t, c)|=+\infty$.

(C) We can identify $\partial D_{i}$ for $i=1,2,3$ by

$$
\begin{aligned}
& \lim _{t \rightarrow t(c, D)-} \Re I(t, c)=+\infty \Longleftrightarrow c(t(c, D)) \in \partial D_{1}, \\
& \lim _{t \rightarrow t(c, D)-} \Re I(t, c)=-\infty \text { and } \lim _{t \rightarrow t(c, D)-} \Im I(t, c)<+\infty \Longleftrightarrow c(t(c, D)) \in \partial D_{2}, \\
& \lim _{t \rightarrow t(c, D)-} \Re I(t, c)=-\infty \text { and } \lim _{t \rightarrow t(c, D)-} \Im I(t, c)=+\infty \Longleftrightarrow c(t(c, D)) \in \partial D_{3} .
\end{aligned}
$$

REMARK 3.7. The results (A) and (B) give a criterion for the geometric impact parameter $t(c, D)$ in terms of the indicator function. It is easy to see that $T$ with the properties (1), (2) is given by

$$
T=\sup \left\{0<t<1\left|\sup _{0 \leq s \leq t}\right| \Re I(s, c) \mid<+\infty\right\} .
$$

Furthermore, since $\partial D=\partial D_{1} \cup \partial D_{2} \cup \partial D_{3}$, we can identify $\partial D_{i}$ according to (C). 
Proof. For a given needle $c(t)$, by Lemma 3.3. we know that there exists a sequence $\left\{v_{n}(x)\right\} \in H^{1}(\Omega)$ which satisfies

$$
\begin{cases}\Delta v_{n}+k^{2} v_{n}=0, & \text { in } \Omega, \\ v_{n}=f_{n}(\cdot, c(t)), & \text { on } \partial \Omega,\end{cases}
$$

and

$$
v_{n} \longrightarrow G(\cdot-c(t)) \quad \text { in } \quad H_{l o c}^{1}\left(\Omega \backslash c_{t}\right) \quad(n \longrightarrow \infty) .
$$

Let $u_{n}(x) \in H^{1}(\Omega \backslash \bar{D})$ satisfy

$$
\begin{cases}\Delta u_{n}+k^{2} u_{n}=0, & \text { in } \Omega \backslash \bar{D}, \\ B_{i} u_{n}=0, & \text { on } \partial D_{i}, \quad i=1,2,3, \\ u_{n}=f_{n}, & \text { on } \partial \Omega .\end{cases}
$$

Then $w_{n}=u_{n}-\left.v_{n}\right|_{\Omega \backslash \bar{D}} \in H^{1}(\Omega \backslash \bar{D})$ satisfies

$$
\begin{cases}\Delta w_{n}+k^{2} w_{n}=0, & \text { in } \Omega \backslash \bar{D}, \\ B_{j} w_{n}=-B_{j} v_{n}, & \text { on } \partial D_{j}, \quad j=1,2,3, \\ w_{n}=0, & \text { on } \partial \Omega .\end{cases}
$$

By Lemma 2.7 and Lemma 3.3 we know that, for $c_{t} \cap \bar{D}=\emptyset$, it follows that

$$
w_{n} \longrightarrow w \quad \text { in } \quad H^{1}(\Omega \backslash \bar{D}), \quad n \longrightarrow \infty
$$

where $w=w(x, c(t))$ satisfies

$$
\begin{cases}\Delta w+k^{2} w=0, & \text { in } \Omega \backslash \bar{D}, \\ B_{j} w=-B_{j} G(\cdot-c(t)), & \text { on } \partial D_{j}, \quad j=1,2,3, \\ w=0, & \text { on } \partial \Omega .\end{cases}
$$

On the other hand, by the calculation in Section 6, we have two kinds of expressions for

$$
\begin{aligned}
\overbrace{\left(\Lambda_{\partial D, \sigma}-\Lambda_{0,0}\right) f_{n}}, f_{n}\rangle, \text { i.e., } & \overbrace{\left(\Lambda_{\partial D, \sigma}-\Lambda_{0,0}\right) f_{n}(\cdot, c(t))}, f_{n}(\cdot, c(t))\rangle \\
= & -\int_{\Omega \backslash \bar{D}}\left(\left|\nabla w_{n}\right|^{2}-k^{2}\left|w_{n}\right|^{2}\right) d x-\int_{D}\left(\left|\nabla v_{n}\right|^{2}-k^{2}\left|v_{n}\right|^{2}\right) d x \\
& +\int_{\partial D_{3}}\left(2 i \sigma v_{n} \tilde{w}_{n}+i \sigma\left|v_{n}\right|^{2}+i \sigma\left|w_{n}\right|^{2}\right) d s \\
& +\int_{\partial D_{1}}\left[\left(v_{n} \frac{\partial \tilde{w}_{n}}{\partial \nu}-\tilde{w}_{n} \frac{\partial v_{n}}{\partial \nu}\right)+\left(v_{n} \frac{\partial \tilde{v}_{n}}{\partial \nu}-\tilde{w}_{n} \frac{\partial w_{n}}{\partial \nu}\right)\right] d s . \\
& \overbrace{\left(\Lambda_{\partial D, \sigma}-\Lambda_{0,0}\right) f_{n}(\cdot, c(t))}, f_{n}(\cdot, c(t))\rangle \\
& \int_{\Omega \backslash \bar{D}}\left(\left|\nabla w_{n}\right|^{2}-k^{2}\left|w_{n}\right|^{2}\right) d x+\int_{D}\left(\left|\nabla v_{n}\right|^{2}-k^{2}\left|v_{n}\right|^{2}\right) d x \\
& -\int_{\partial D_{2}}\left[\tilde{u}_{n} \frac{\partial v_{n}}{\partial \nu}+u_{n} \frac{\partial \tilde{v}_{n}}{\partial \nu}\right] d s-\int_{\partial D_{3}}\left[\tilde{u}_{n} \frac{\partial v_{n}}{\partial \nu}+u_{n} \frac{\partial \tilde{v}_{n}}{\partial \nu}-i \sigma\left|u_{n}\right|^{2}\right] d s .
\end{aligned}
$$


Let $n$ tend to infinity in (3.7). Then, by (3.5), we have

$$
\begin{aligned}
-I(t, c) & =\int_{D}\left\{|\nabla G(\cdot-c(t))|^{2}-k^{2}|G(\cdot-c(t))|^{2}\right\} d x \\
& +\int_{\Omega \backslash \bar{D}}\left\{|\nabla w|^{2}-k^{2}|w|^{2}\right\} d x \\
& -i \int_{\partial D_{3}} \sigma(x)\left\{|G(\cdot-c(t))|^{2}+|w|^{2}\right\} d s-2 i \int_{\partial D_{3}} \sigma(x) \tilde{w} G d s \\
& -\int_{\partial D_{1}}\left[\left(G \frac{\partial \tilde{w}}{\partial \nu}-\tilde{w} \frac{\partial G}{\partial \nu}\right)+\left(G \frac{\partial \tilde{G}}{\partial \nu}-\tilde{w} \frac{\partial w}{\partial \nu}\right)\right] d s .
\end{aligned}
$$

We first prove (A). If $t(c, D)=1$, then, by the definition of $t(c, D)$, we know that $c(t)$ does not touch $\bar{D}$, i.e. $c(t) \in \Omega \backslash \bar{D}$ for $0 \leq t \leq 1$. It is easy to verify that

$$
\sup _{0 \leq t \leq 1}|\Re I(t, c)|<+\infty .
$$

The converse is true if we prove (B). So, let's go to the proof of $(\mathrm{B})$. Let $t(c, D)<$ 1 and $x_{0}=c(t(c, D)) \in \partial D$. Then, it is easy to see that we only have to prove $\lim _{t \rightarrow t(c, D)-}|\Re I(t, c)|=+\infty$. This is included in the proof of $(\mathrm{C})$. So the rest of the argument is devoted to the proof of $(\mathrm{C})$.

Likewise before, since $c(t) \in \Omega \backslash \bar{D}(0 \leq t<t(c, D))$, we have $\sup _{0 \leq s \leq t}|\Re I(s, c)|<+\infty$ for $0 \leq t<t(c, D)$. Now observe that

$$
\begin{aligned}
- & \Re I(t, c)=\int_{D}\left[|\nabla G(\cdot-c(t))|^{2}-k^{2}|G(\cdot-c(t))|^{2}\right] d x \\
+ & \int_{\Omega \backslash \bar{D}}\left(|\nabla w|^{2}-k^{2}|w|^{2}\right) d x+2 \int_{\partial D_{3}} \sigma(x) \Im(\tilde{w} G(\cdot-c(t))) d s \\
& -\Re\left[\int_{\partial D_{1}}\left(G \frac{\partial \tilde{w}}{\partial \nu}-\tilde{w} \frac{\partial G}{\partial \nu}+G \frac{\partial \tilde{G}}{\partial \nu}-\tilde{w} \frac{\partial w}{\partial \nu}\right) d s\right] \\
\geq & \int_{D}|\nabla G(\cdot-c(t))|^{2} d x-k^{2} \int_{D}|G(\cdot-c(t))|^{2} d x-k^{2} \int_{\Omega \backslash \bar{D}}|w|^{2} d x \\
+ & 2 \int_{\partial D_{3}} \sigma(x) \Im(\tilde{w} G(\cdot-c(t))) d s \\
& -\Re\left[\int_{\partial D_{1}}\left(G \frac{\partial \tilde{w}}{\partial \nu}-\tilde{w} \frac{\partial G}{\partial \nu}+G \frac{\partial \tilde{G}}{\partial \nu}-\tilde{w} \frac{\partial w}{\partial \nu}\right) d s\right] .
\end{aligned}
$$

According to the result of singularity analysis about $w\left(x, x_{0}\right)$ and $G\left(x-x_{0}\right)$ for $x_{0} \in \partial D$ given in Theorem 5.1 and Theorem 5.2 below, we have from (3.10),

$$
\lim _{t \longrightarrow t\left(c, D_{j}\right)-} \Re(I(t, c))=-\infty
$$

if $j=2,3$. On the other hand, consider the real part of the limit of (3.8) as $n \rightarrow$ $\infty$. It is easy to find that the real part will tend to $+\infty$ when $c(t) \rightarrow \partial D_{1}$, since $\int_{D}|\nabla G(\cdot-c(t))|^{2} d x$ will blow up, while the integrals on the boundary are clearly bounded and the $L^{2}$ integral of $w$ is bounded by Theorem [5.1. These facts imply that we can 
distinguish the sound-soft boundary $D_{1}$ from the other two kinds of boundaries (soundhard and Robin-type). Now we want to distinguish $\partial D_{2}$ and $\partial D_{3}$ furthermore. For this purpose, we need to consider the imaginary part of (3.8). In fact, it yields from (3.7) that

$$
\left.\Im(\overbrace{\left\langle\left(\Lambda_{\partial D, \sigma}-\Lambda_{0,0}\right) f_{n}\right.}, f_{n}\rangle\right)=\int_{\partial D_{3}} \sigma(x)\left|u_{n}\right|^{2} d s .
$$

Now we estimate the behavior of the imaginary part of the indicator function. Recalling our previous notation, we get

$$
\begin{aligned}
& \left.\lim _{n \rightarrow \infty} \Im(\overbrace{\left(\Lambda_{\partial D, \sigma}-\Lambda_{0,0}\right) f_{n}}, f_{n}\rangle\right)=\lim _{n \rightarrow \infty} \int_{\partial D_{3}} \sigma(x)\left|u_{n}\right|^{2} d s \\
= & \lim _{n \rightarrow \infty} \int_{\partial D_{3}} \sigma(x)\left|v_{n}+w_{n}\right|^{2} d s \\
= & \int_{\partial D_{3}} \sigma(x)|(G(x-c(t)))+w(x, c(t))|^{2} d s,
\end{aligned}
$$

where $w$ is the function defined by (3.6). According to the singularity analysis in section 5 and section 6 , we know that $|G(x-c(t))+w(x, c(t))|$ is estimated by $|G(x-c(t))|$. Hence, from (3.12) and the estimate for $G$ in section 5 , we have

$$
\begin{aligned}
\lim _{t \rightarrow t\left(c, D_{3}\right)-} \Im I(t, c) & =\lim _{t \rightarrow t\left(c, D_{3}\right)-} \int_{\partial D_{3}} \sigma(x)|(G(x-c(t)))+w(x, c(t))|^{2} d s=+\infty \\
\lim _{t \rightarrow t\left(c, D_{2}\right)-} \Im I(t, c) & =\lim _{t \rightarrow t\left(c, D_{2}\right)-} \int_{\partial D_{3}} \sigma(x)|G(x-c(t))+w(x, c(t))|^{2} d s<\infty .
\end{aligned}
$$

Since $D_{2}$ and $D_{3}$ are separated, these behaviors of $\Im I(t, c)$ enable us to distinguish $\partial D_{3}$ and $\partial D_{2}$.

The proof is complete.

Now we give the reconstruction procedure for the shape and type of each obstacle. It can be realized by the following steps:

- Calculate the Dirichlet-to-Neumann map $\Lambda_{\sigma, D}$ from the far-field patterns $u^{\infty}(d, \theta), d, \theta \in S^{2}$.

- For any given needle $c(t)$, calculate the sequences $v_{n}$ and $f_{n}(\cdot, c)$.

- Calculate $\langle\overbrace{\left(\Lambda_{\partial D, \sigma}-\Lambda_{0,0}\right) f_{n}(\cdot, c(t))}, f_{n}(\cdot, c(t))\rangle$.

- Calculate $I(t, c)$ and

$$
\begin{aligned}
\partial D_{1}= & \left\{c\left(t_{0}\right)\left|\sup _{0 \leq s \leq t}\right| \Re I(s, c) \mid<+\infty \text { for } 0 \leq t<t_{0}, \lim _{t \rightarrow t_{0}-} \Re I(t, c)=+\infty\right\}, \\
\partial D_{2}= & \left\{c\left(t_{0}\right)\left|\sup _{0 \leq s \leq t}\right| \Re I(s, c)\left|, \sup _{0 \leq s \leq t}\right| \Im I(s, c) \mid<+\infty \quad \text { for } 0 \leq t<t_{0},\right. \\
& \left.\lim _{t \rightarrow t_{0}-} \Re I(t, c)=-\infty \text { and } \lim _{t \rightarrow t_{0}-} \Im I(t, c)<+\infty\right\}, \\
\partial D_{3}= & \left\{c\left(t_{0}\right)\left|\sup _{0 \leq s \leq t}\right| \Re I(s, c)\left|, \sup _{0 \leq s \leq t}\right| \Im I(s, c) \mid<+\infty \text { for } 0 \leq t<t_{0},\right. \\
& \left.\lim _{t \rightarrow t_{0}-} \Re I(t, c)=-\infty \text { and } \lim _{t \rightarrow t_{0}-} \Im I(t, c)=+\infty\right\} .
\end{aligned}
$$

The rest of the proof of Theorem 1.1 is to reconstruct the boundary impedance on $D_{3}$. This will be given in the next section. 
4. Moment method for determining $\sigma(x)$. In this section, we reconstruct the boundary impedance $\sigma(x)$. Since in the previous section, we have reconstructed $\partial D$ from the far-field patterns $u^{\infty}(d, \theta), d, \theta \in S^{2}$, therefore in this section we assume that $\partial D=\partial D_{1} \cup \partial D_{2} \cup \partial D_{3}$ is known.

Consider the boundary value problem

$$
\begin{cases}\Delta u+k^{2} u=0, & \text { in } \Omega \backslash \bar{D}, \\ B_{j} u(x)=0, & \text { on } \partial D_{j}, \quad j=1,2, \\ \frac{\partial u}{\partial \nu}+i \sigma(x) u=0, & \text { on } \partial D_{3}, \\ u(x)=f, & \text { on } \partial \Omega\end{cases}
$$

for a given $f(x) \in H^{1 / 2}(\partial \Omega)$.

Lemma 4.1. Suppose that $u_{j}(x) \in H^{1}(\Omega \backslash \bar{D}), j=1,2, \cdots$ satisfy (4.1) with $f=f_{j}$. Put $\phi_{j}(x)=\left.u_{j}(x)\right|_{\partial D_{3}}$. If

$$
\overline{\operatorname{span}\left\{f_{j}(x)\right\}}=H^{1 / 2}(\partial \Omega),
$$

then we have

$$
\overline{\operatorname{span}\left\{\phi_{j}(x)\right\}}=H^{1 / 2}\left(\partial D_{3}\right) .
$$

Proof. Assume that $f(x) \in H^{-1 / 2}\left(\partial D_{3}\right)$ satisfies

$$
\int_{\partial D_{3}} \phi_{j} \tilde{f} d s=0, \quad j=1,2, \cdots
$$

We want to prove that $f(x)=0$. Here $\int_{\partial D_{3}} \phi_{j} \tilde{f} d s$ denotes the pairing $\left\langle\tilde{f}, \phi_{j}\right\rangle$ between $H^{-1 / 2}\left(\partial D_{3}\right)$ and $H^{1 / 2}\left(\partial D_{3}\right)$.

Consider the following boundary value problem:

$$
\begin{cases}\Delta v+k^{2} v=0, & \text { in } \Omega \backslash \bar{D}, \\ B_{j} v(x)=0, & \text { on } \partial D_{j}, \quad j=1,2, \\ \frac{\partial v}{\partial \nu}+i \sigma(x) v=\tilde{f}, & \text { on } \partial D_{3}, \\ v=0, & \text { on } \partial \Omega .\end{cases}
$$

Since $\sigma(x)>0$, similar to the proof of Lemma 2.3, we know there exists a unique solution $v \in H^{1}(\Omega \backslash \bar{D})$ for (4.4).

By Green's formula, we know that

$$
\begin{aligned}
0 & =\int_{\Omega \backslash \bar{D}}\left(v \Delta u_{j}-u_{j} \Delta v\right) d x \\
& =\int_{\partial \Omega}\left(\frac{\partial u_{j}}{\partial \nu_{1}} v-\frac{\partial v}{\partial \nu_{1}} u_{j}\right)+\int_{\partial D}\left(\frac{\partial u_{j}}{\partial \nu_{1}} v-\frac{\partial v}{\partial \nu_{1}} u_{j}\right),
\end{aligned}
$$

where $\nu_{1}$ is the outward normal of the domain $\Omega \backslash \bar{D}$.

Noticing $\nu_{1}=-\nu$ on $\partial D$ and $\left.v\right|_{\partial \Omega}=0$, we have

$$
\int_{\partial \Omega} \frac{\partial v}{\partial \nu} u_{j} d s=\int_{\partial D_{3}}\left(i \sigma v u_{j}+\frac{\partial v}{\partial \nu} u_{j}\right) d s
$$

due to the boundary conditions on $\partial D_{1}$ and $\partial D_{2}$. Therefore, it follows that

$$
\int_{\partial \Omega} f_{j} \frac{\partial v}{\partial \nu} d s=\int_{\partial D_{3}} \phi_{j} \tilde{f} d s=0, \quad j=1,2, \cdots .
$$


Since $\overline{\operatorname{span}\left\{f_{j}(x)\right\}}=H^{1 / 2}(\partial \Omega)$, we obtain

$$
\left.\frac{\partial v}{\partial \nu}\right|_{\partial \Omega}=0
$$

By the uniqueness of the Cauchy problem for the Helmholtz equations in the domain $\Omega \backslash \bar{D}$, we have $v(x)=0$ in $\Omega \backslash \bar{D}$. Then by (4.4), we know that $f(x)=0$. The proof is complete.

On the other hand, we can obtain $\left.u_{j}\right|_{\partial D}$ and $\left.\frac{\partial u_{j}}{\partial \nu}\right|_{\partial D}$ by solving the following Cauchy problem:

$$
\begin{cases}\Delta u_{j}+k^{2} u_{j}=0, & \text { in } \Omega \backslash \bar{D} \\ u_{j}=f_{j}, \frac{\partial u_{j}}{\partial \nu}=\Lambda_{\partial D, \sigma} f_{j}, & \text { on } \partial \Omega\end{cases}
$$

for a given $f_{j}(x)$; hence both $\left.u_{j}\right|_{\partial D_{3}}$ and $\left.\frac{\partial u_{j}}{\partial \nu}\right|_{\partial D_{3}}$ are obtained.

Now, by integrating the Robin-type boundary condition over $\partial D_{3}$, we have that the impedance $\sigma(x)$ satisfies

$$
\int_{\partial D_{3}} i \sigma(x) u_{j} d s=-\int_{\partial D_{3}} \frac{\partial u_{j}}{\partial \nu} d s, \quad j=1,2, \cdots .
$$

Here note that $\operatorname{span}\left\{\left.u_{j}\right|_{\partial D_{3}}\right\}$ is dense in $H^{1 / 2}\left(\partial D_{3}\right)$ by Lemma 4.1 ; hence $\sigma(x)$ can be determined uniquely from this moment problem.

Now the recovery of the impedance $\sigma(x)$ can be realized by the following steps:

- Choose $f_{j}, j=1,2, \cdots$ such that $\overline{\operatorname{span}\left\{f_{j}\right\}_{j=1}^{\infty}}=H^{1 / 2}(\partial \Omega)$.

- For every $f_{j}$, solve the Cauchy problem (4.6) and obtain $\left.u_{j}\right|_{\partial D_{3}}$ and $\left.\frac{\partial u_{j}}{\partial \nu}\right|_{\partial D_{3}}$.

- Solve the moment problem (4.7) to get $\sigma(x)$.

5. Some estimates. In this section we give the proof of Lemma 2.7 and an estimate of $\|w\|_{L^{2}(\Omega \backslash \bar{D})}$.

Proof of Lemma 2.7. Let $p(x)=u(x)-\left.u_{1}(x)\right|_{\Omega \backslash \bar{D}}$. It is easy to verify that $p(x)$ satisfies

$$
\begin{cases}\Delta p+k^{2} p=0, & \text { in } \Omega \backslash \bar{D}, \\ B_{j} p=-B_{j} u_{1}, & \text { on } \partial D_{j}, j=1,2,3, \\ p(x)=0, & \text { on } \partial \Omega .\end{cases}
$$

By the well-posedness of the boundary value problem (5.1), we know that the solution $p(x) \in H^{1}(\Omega \backslash \bar{D})$ depends continuously on the boundary data on $\partial D=\partial D_{1} \cup \partial D_{2} \cup \partial D_{3}$. Therefore, there exists a constant $C>0$ such that

$$
\|p\|_{H^{1}(\Omega \backslash \bar{D})} \leq C\left\{\left\|B_{1} u_{1}\right\|_{H^{1 / 2}\left(\partial D_{1}\right)}+\left\|B_{2} u_{1}\right\|_{H^{-1 / 2}\left(\partial D_{2}\right)}+\left\|B_{3} u_{1}\right\|_{H^{-1 / 2}\left(\partial D_{3}\right)}\right\} .
$$

On the other hand, the trace theorem for $u_{1}(x)$ on the domain $D$ yields

$$
\left\|B_{1} u_{1}\right\|_{H^{1 / 2}\left(\partial D_{1}\right)}+\left\|B_{2} u_{1}\right\|_{H^{-1 / 2}\left(\partial D_{2}\right)}+\left\|B_{3} u_{1}\right\|_{H^{-1 / 2}\left(\partial D_{3}\right)} \leq C\left\|u_{1}\right\|_{H^{1}(D)}
$$

due to $0<\sigma(x)<\sigma_{0}$, and the proof is complete.

Theorem 5.1. There exists a constant $C$ independent of $D$ such that

$$
\|w\|_{L^{2}(\Omega \backslash \bar{D})} \leq C .
$$


Proof. We adapt the proof of [12] to our case. First we define a function $v(x)$ by

$$
\begin{cases}\Delta v+k^{2} v=w, & \text { in } \Omega \backslash \bar{D}, \\ B_{j} v=0, & \text { on } \partial D_{j}, \quad j=1,2, \\ \frac{\partial v}{\partial \nu}+\widetilde{i \sigma} v=0, & \text { on } \partial D_{3}, \\ v(x)=0, & \text { on } \partial \Omega\end{cases}
$$

Then, from the well-posedness of this boundary problem, we have

$$
\|v\|_{H^{2}(\Omega \backslash \bar{D})} \leq C\|w\|_{L^{2}(\Omega \backslash \bar{D})} \text {. }
$$

Since $\Omega \backslash \bar{D}$ is a domain in $R^{3}$ with $C^{2}$ boundary, by the Sobolev embedding theorems, we know that $H^{2}(\Omega \backslash \bar{D})$ can be embedded into $B^{1 / 2}(\Omega \backslash \bar{D})$ (Hölder space with exponent $1 / 2)$ and $\|v\|_{B^{1 / 2}} \leq C\|v\|_{H^{2}}$. So we have

$$
\|v\|_{B^{1 / 2}} \leq C\|w\|_{L^{2}} .
$$

From this inequality, we know that

$$
\left\{\begin{array}{l}
|v(x)-v(y)| \leq C|x-y|^{1 / 2}\|w\|_{L^{2}(\Omega \backslash \bar{D})}, \quad x, y \in \Omega \backslash \bar{D} \\
\|v\|_{L^{\infty}(\Omega \backslash \bar{D})} \leq C\|w\|_{L^{2}(\Omega \backslash \bar{D})} .
\end{array}\right.
$$

Recalling the definition of weak solutions $w$ and $v$ to (3.6) and (5.3) respectively, by the Green formula and the boundary conditions for $v, w$, we have

$$
\begin{aligned}
0= & \int_{\Omega \backslash \bar{D}}\left(\Delta+k^{2}\right) \tilde{w}(x) v(x) d x \\
= & -\int_{\partial D} v \frac{\partial \tilde{w}}{\partial \nu} d s-\int_{\Omega \backslash \bar{D}}\left(\nabla \tilde{w}(x) \nabla v(x)-k^{2} \tilde{w}(x) v(x)\right) d x \\
= & \int_{\partial D_{2}} v \frac{\partial \tilde{G}(x-c(t))}{\partial \nu} d s+\int_{\partial D_{3}} v\left[\tilde{w} \tilde{\sigma}+\left(\frac{\partial}{\partial \nu}+\tilde{i \sigma}\right) \tilde{G}\right] d s \\
& -\int_{\Omega \backslash \bar{D}}\left(\nabla \tilde{w}(x) \nabla v(x)-k^{2} \tilde{w}(x) v(x)\right) d x
\end{aligned}
$$

which yields

$$
\begin{aligned}
& \int_{\Omega \backslash \bar{D}}|w(x)|^{2} d x=\int_{\Omega \backslash \bar{D}}\left(\Delta+k^{2}\right) v \tilde{w} d x \\
= & \int_{\partial D_{1}} \tilde{G} \frac{\partial v}{\partial \nu} d s+\int_{\partial D_{3}} \tilde{w} \tilde{\sigma} v d s-\int_{\Omega \backslash \bar{D}}\left(\nabla \tilde{w}(x) \cdot \nabla v(x)-k^{2} \tilde{w}(x) v(x)\right) d x \\
= & \int_{\partial D_{1}} \tilde{G} \frac{\partial v}{\partial \nu} d s-\int_{\partial D_{2}} v \frac{\partial \tilde{G}}{\partial \nu} d s-\int_{\partial D_{3}} v\left(\frac{\partial}{\partial \nu}+\tilde{i \sigma}\right) \tilde{G} d s \\
= & \int_{\partial D_{1}} \tilde{G} \frac{\partial v}{\partial \nu} d s-\int_{\partial D_{3}} v \tilde{i} \tilde{G} d s-v(c(t))\left[\int_{\partial D_{2}}+\int_{\partial D_{3}}\right] \frac{\partial \tilde{G}}{\partial \nu} d s \\
& -\left[\int_{\partial D_{2}}+\int_{\partial D_{3}}\right](v(x)-v(c(t))) \frac{\partial \tilde{G}(x-c(t))}{\partial \nu} d s .
\end{aligned}
$$

On the other hand, if $y \notin \bar{D}_{j}$, we have

$$
\int_{\partial D_{j}} \frac{\partial}{\partial \nu} \tilde{G}(x-y) d s+k^{2} \int_{D_{j}} \tilde{G}(x-y) d x=\int_{D_{j}}\left(\Delta+k^{2}\right) \tilde{G}(x-y) d x=0 .
$$


Therefore (5.6) leads to

$$
\begin{aligned}
& \|w\|_{L^{2}(\Omega \backslash \bar{D})}^{2} \\
= & \int_{\partial D_{1}} \tilde{G} \frac{\partial v}{\partial \nu} d s-\int_{\partial D_{3}} v \widetilde{i \sigma G} d s+k^{2} v(c(t))\left(\int_{D_{2}}+\int_{D_{3}}\right) \tilde{G}(x-c(t)) d x \\
& -\left(\int_{\partial D_{2}}+\int_{\partial D_{3}}\right)(v(x)-v(c(t))) \frac{\partial \tilde{G}(x-c(t))}{\partial \nu} d s .
\end{aligned}
$$

On the one hand, (5.5) tells us

$$
\frac{|v(x)-v(y)|}{|x-y|} \leq C \frac{1}{|x-y|^{1 / 2}}\|w\|_{L^{2}(\Omega \backslash \bar{D})},
$$

which implies

$$
\left|\int_{\partial D_{1}} \tilde{G} \frac{\partial v}{\partial \nu} d s\right| \leq C\|w\|_{L^{2}(\Omega \backslash \bar{D})} \int_{\partial D_{1}}|\tilde{G}(x-c(t))| \frac{1}{|x-c(t)|^{1 / 2}} d s \leq C\|w\|_{L^{2}(\Omega \backslash \bar{D})}
$$

as $c(t) \longrightarrow \partial D_{1}$. On the other hand, the integrals $\int_{\partial D}|\tilde{G}(x-c(t))| d s, \int_{D}|\tilde{G}(x-c(t))| d x$ and $\int_{\partial D}|x-c(t)|^{1 / 2}\left|\frac{\partial}{\partial \nu} \tilde{G}(x-c(t))\right| d s$ are bounded as $c(t) \longrightarrow \partial D$. Therefore by (5.5) and (5.7), we have

$$
\|w\|_{L^{2}(\Omega \backslash \bar{D})}^{2} \leq C\|w\|_{L^{2}(\Omega \backslash \bar{D})} .
$$

The proof is complete.

Theorem 5.2. Assume $x_{0} \in \partial D$ and $c(t) \in(\Omega \backslash \bar{D}) \cap \partial B\left(x_{0}, \delta\right)$ for some $\delta>0$, where $B\left(x_{0}, \delta\right)$ is an open ball centered at $x_{0}$ with radius $\delta$, then there exists some constant $C>0$ such that for $\delta$ small enough the following estimates hold:

$$
\begin{aligned}
& \int_{D}|\nabla G(x-c(t))|^{2} d x \geq \frac{C}{\delta}, \quad \int_{D}|G(x-c(t))|^{2} d x \leq C, \\
& \int_{\partial D}|G(x-c(t))|^{2} d s \leq C|\ln \delta|, \quad \int_{\partial D}|w(x, c(t))|^{2} d s \leq C \int_{\partial D}|G(x-c(t))|^{2} d s,
\end{aligned}
$$

where $D$ should be $D_{j}$ for $j=1,2,3$ in the former three estimates and for $j=2,3$ in the fourth estimate; the constants $C>0$ may be different.

Proof. Except the fourth estimate, the proofs of the estimates are given in [1]. For the reader's convenience we repeat them. Denote the tangent plane of $\partial D$ at point $x_{0}$ by $T\left(x_{0}, \partial D\right)$. From the expressions of Green's function, we have

$$
|\nabla G(x-c(t))|^{2}=O\left(\frac{1}{|x-c(t)|^{4}}\right), \quad|G(x-c(t))|^{2}=O\left(\frac{1}{|x-c(t)|^{2}}\right) .
$$

We have that, for $\delta$ small enough,

$$
\begin{aligned}
\int_{D} \frac{1}{|x-c(t)|^{4}} \mid d x & \geq \int_{D \cap B\left(x_{0}, \delta\right)} \frac{1}{|x-c(t)|^{4}} \mid d x \geq \int_{D \cap B\left(x_{0}, \delta\right)} \frac{1}{(2 \delta)^{4}} d x \\
& =\frac{1}{(2 \delta)^{4}} \int_{D \cap B\left(x_{0}, \delta\right)} d x \geq \frac{1}{(2 \delta)^{4}} \frac{1}{4} \int_{B\left(x_{0}, \delta\right)} d x=\frac{C}{\delta} .
\end{aligned}
$$

Hence we have obtained the first estimate. The second estimate is obvious. 
On the other hand, let $c^{\prime}(t) \in \Omega \backslash \bar{D}$ satisfy

$$
c^{\prime}(t) \in \partial B\left(x_{0}, \delta\right), \quad c^{\prime}(t)-x_{0} \text { is perpendicular to } T\left(x_{0}, \partial D\right) .
$$

Then,

where

$$
\int_{\partial D}|G(x-c(t))|^{2} d x \leq C\left(\int_{\partial D 1}+\int_{\partial D 2}\right) \frac{1}{|x-c(t)|^{2}} d x
$$

$$
\begin{aligned}
& \partial D 1:=\partial D \cap\left\{x \in R^{3}|| x-\left.c^{\prime}(t)\right|^{2} \geq \frac{1}{|\ln \delta|}\right\}, \\
& \partial D 2:=\partial D \cap\left\{x \in R^{3}|| x-\left.c^{\prime}(t)\right|^{2} \leq \frac{1}{|\ln \delta|}\right\} .
\end{aligned}
$$

Since $\frac{1}{2} \frac{1}{|\ln \delta|^{1 / 2}}-2 \delta \geq 0$ for small enough $\delta$ and

$$
\left|c(t)-c^{\prime}(t)\right| \leq 2 \delta, \quad|x-c(t)| \geq \frac{1}{2} \frac{1}{|\ln \delta|^{1 / 2}} \quad\left(x \in \partial D_{1}\right),
$$

we have

$$
\int_{\partial D 1} \frac{1}{|x-c(t)|^{2}} d s \leq 4|\ln \delta| \int_{\partial D 1} d s \leq C|\ln \delta| .
$$

For the second integral, since

$$
\partial D 2^{\prime}=\left\{x \in R^{3}\left|x \in T\left(x_{0}, \partial D\right),\right| x-\left.x_{0}\right|^{2} \leq \frac{2}{|\ln \delta|}-\delta^{2}\right\}
$$

approximates $\partial D 2$ for small $\delta>0$, we know that

$$
\begin{aligned}
\int_{\partial D 2} \frac{1}{|x-c(t)|^{2}} d s & \leq 2 \int_{\partial D 2^{\prime}} \frac{1}{|x-c(t)|^{2}} d s=\int_{\partial D 2^{\prime}} \frac{2}{\left|x-x_{0}\right|^{2}+\delta^{2}} d s \\
& =2 \int_{0}^{2 \pi} \int_{0}^{\sqrt{2|\ln \delta|^{-1}-\delta^{2}}} \frac{r d r d \theta}{r^{2}+\delta^{2}} \\
& =4 \pi\left(|\ln \delta|+\frac{1}{2} \ln 2-\frac{1}{2} \ln (\mid \ln \delta) \mid\right) \leq C|\ln \delta|
\end{aligned}
$$

for $\delta>0$ small enough. Then the third estimate follows from (5.10)-(5.11).

The fourth estimate will be given in the next section. The proof is complete.

\section{Singularity analysis.}

6.1. Expression of $\Lambda_{\partial D, \sigma}-\Lambda_{0,0}$. Here we prove (3.7). Let $v(x) \in H^{1}(\Omega \backslash \bar{D})$. From the definition of the weak solution of $u_{n}$ to (3.4), we have

$$
0=\int_{\partial \Omega} v \frac{\partial u_{n}}{\partial \nu_{1}} d s+\int_{\partial D} v \frac{\partial u_{n}}{\partial \nu_{1}} d s-\int_{\Omega \backslash \bar{D}}\left(\nabla u_{n} \nabla v-k^{2} v u_{n}\right) d x .
$$

Hence, recalling the boundary condition of $u_{n}$, we get

$$
\int_{\partial \Omega} v \frac{\partial u_{n}}{\partial \nu_{1}} d s=\int_{\Omega \backslash \bar{D}}\left(\nabla u_{n} \nabla v-k^{2} v u_{n}\right) d x+\int_{\partial D_{1}} \frac{\partial u_{n}}{\partial \nu} v d s-\int_{\partial D_{3}} i \sigma u_{n} v d s .
$$

Firstly, taking $v=\tilde{w}_{n}$ in this expression, we get that

$$
0=\int_{\Omega \backslash \bar{D}}\left(\nabla u_{n} \nabla \tilde{w}_{n}-k^{2} \tilde{w}_{n} u_{n}\right) d x+\int_{\partial D_{1}} \frac{\partial u_{n}}{\partial \nu} \tilde{w}_{n} d s-\int_{\partial D_{3}} i \sigma u_{n} \tilde{w}_{n} d s .
$$


due to $\left.w_{n}\right|_{\Omega}=0$. On the other hand, by taking $v=\tilde{v}_{n}$ also in the above expression, we have

$$
\left\langle\Lambda_{\partial D, \sigma} f_{n}, \tilde{f}_{n}\right\rangle=\int_{\Omega \backslash \bar{D}}\left(\nabla u_{n} \nabla \tilde{v}_{n}-k^{2} \tilde{v}_{n} u_{n}\right) d x+\int_{\partial D_{1}} \frac{\partial u_{n}}{\partial \nu} \tilde{v}_{n} d s-\int_{\partial D_{3}} i \sigma u_{n} \tilde{v}_{n} d s .
$$

Analogously, we have

$$
\left\langle\Lambda_{0,0} f_{n}, \tilde{f}_{n}\right\rangle=\int_{\partial \Omega} \frac{\partial v_{n}}{\partial \nu_{1}} \tilde{v}_{n} d s=\int_{\Omega}\left(\nabla v_{n} \nabla \tilde{v}_{n}-k^{2} v_{n} \tilde{v}_{n}\right) d x
$$

From the above expression, we get that

$$
\begin{aligned}
\overbrace{\left\langle\left(\Lambda_{\partial D, \sigma}-\Lambda_{0,0}\right) f_{n}, \tilde{f}_{n}\right\rangle}= & \int_{\Omega \backslash \bar{D}}\left(\nabla \tilde{w}_{n} \nabla v_{n}-k^{2} \tilde{w}_{n} v_{n}\right) d x+\int_{\partial D_{1}} \frac{\partial \tilde{u}_{n}}{\partial \nu} v_{n} d s \\
& -\int_{D}\left(\left|\nabla v_{n}\right|^{2}-k^{2}\left|v_{n}\right|^{2}\right) d x-\int_{\partial D_{3}} \tilde{i \sigma} \tilde{u}_{n} v_{n} d s .
\end{aligned}
$$

Recall $w_{n}=u_{n}-v_{n},(6.2)$ and consider

$$
\begin{aligned}
J_{n}: & =\int_{\Omega \backslash \bar{D}}\left(\nabla w_{n} \nabla \tilde{w}_{n}-k^{2} w_{n} \tilde{w}_{n}\right) d x-\int_{\partial D_{3}} i \sigma w_{n} \tilde{w}_{n} d s+\int_{\partial D_{1}} \tilde{w}_{n} \frac{\partial w_{n}}{\partial \nu} d s \\
& =-\int_{\Omega \backslash \bar{D}}\left(\nabla v_{n} \nabla \tilde{w}_{n}-k^{2} v_{n} \tilde{w}_{n}\right) d x+\int_{\partial D_{3}} i \sigma v_{n} \tilde{w}_{n} d s-\int_{\partial D_{1}} \tilde{w}_{n} \frac{\partial v_{n}}{\partial \nu} d s .
\end{aligned}
$$

Then, we get

$$
\int_{\Omega \backslash \bar{D}}\left(\nabla v_{n} \nabla \tilde{w}_{n}-k^{2} v_{n} \tilde{w}_{n}\right) d x=-J_{n}+\int_{\partial D_{3}} i \sigma v_{n} \tilde{w}_{n} d s-\int_{\partial D_{1}} \tilde{w}_{n} \frac{\partial v_{n}}{\partial \nu} d s
$$

Inserting this expression into (6.5), and recalling (6.2), (6.3) leads to

$$
\begin{aligned}
& \overbrace{\left(\Lambda_{\partial D, \sigma}-\Lambda_{0,0}\right) f_{n}}, f_{n}\rangle \\
& =-J_{n}+\int_{\partial D_{3}} i \sigma v_{n} \tilde{w}_{n} d s-\int_{\partial D_{1}} \tilde{w}_{n} \frac{\partial v_{n}}{\partial \nu} d s-\int_{D}\left(\left|\nabla v_{n}\right|^{2}-k^{2}\left|v_{n}\right|^{2}\right) d x \\
& +\int_{\partial D_{1}} \frac{\partial \tilde{u}_{n}}{\partial \nu} v_{n} d s-\int_{\partial D_{3}} \tilde{i \sigma} \tilde{u}_{n} v_{n} d s \\
& =-\int_{\Omega \backslash \bar{D}}\left(\nabla w_{n} \nabla \tilde{w}_{n}-k^{2} w_{n} \tilde{w}_{n}\right) d x+\int_{\partial D_{3}} i \sigma w_{n} \tilde{w}_{n} d s-\int_{\partial D_{1}} \tilde{w}_{n} \frac{\partial w_{n}}{\partial \nu} d s \\
& +\int_{\partial D_{3}} i \sigma v_{n} \tilde{w}_{n} d s-\int_{\partial D_{1}} \tilde{w}_{n} \frac{\partial v_{n}}{\partial \nu} d s-\int_{D}\left(\left|\nabla v_{n}\right|^{2}-k^{2}\left|v_{n}\right|^{2}\right) d x \\
& +\int_{\partial D_{1}} \frac{\partial\left(\tilde{v}_{n}+\tilde{w}_{n}\right)}{\partial \nu} v_{n} d s-\int_{\partial D_{3}} \tilde{i \sigma}\left(\tilde{v}_{n}+\tilde{w}_{n}\right) v_{n} d s \\
& =-\int_{\Omega \backslash \bar{D}}\left(\left|\nabla w_{n}\right|^{2}-k^{2}\left|w_{n}\right|^{2}\right) d x-\int_{D}\left(\left|\nabla v_{n}\right|^{2}-k^{2}\left|v_{n}\right|^{2}\right) d x \\
& +\int_{\partial D_{3}}\left(2 i \sigma v_{n} \tilde{w}_{n}+i \sigma\left|v_{n}\right|^{2}+i \sigma\left|w_{n}\right|^{2}\right) d s \\
& +\int_{\partial D_{1}}\left[\left(v_{n} \frac{\partial \tilde{w}_{n}}{\partial \nu}-\tilde{w}_{n} \frac{\partial v_{n}}{\partial \nu}\right)+\left(v_{n} \frac{\partial \tilde{v}_{n}}{\partial \nu}-\tilde{w}_{n} \frac{\partial w_{n}}{\partial \nu}\right)\right] d s .
\end{aligned}
$$


This expression will be used to identify $\partial D_{1}$ from $\partial D$. Now we prove the other expression (3.8) for $\langle\overbrace{\left(\Lambda_{\partial D, \sigma}-\Lambda_{0,0}\right) f_{n}}, f_{n}\rangle$, which applies the value of $v_{n}$ and $w_{n}$ on $\partial D_{2}$ and $\partial D_{3}$. This expression will be applied furthermore to distinguish $\partial D_{2}$ and $\partial D_{3}$ from $\partial D$.

By a straightforward calculation, we get

$$
\begin{aligned}
\int_{\Omega \backslash \bar{D}}\left|\nabla\left(u_{n}-v_{n}\right)\right|^{2} d x= & \int_{\partial \Omega} u_{n} \frac{\partial\left(\tilde{u}_{n}-\tilde{v}_{n}\right)}{\partial \nu} d s-\int_{\partial D} u_{n} \frac{\partial\left(\tilde{u}_{n}-\tilde{v}_{n}\right)}{\partial \nu} d s \\
& +\int_{\Omega \backslash \bar{D}} k^{2} u_{n}\left(\tilde{u}_{n}-\tilde{v}_{n}\right) d x-\int_{\Omega \backslash \bar{D}} \nabla v_{n} \cdot \nabla\left(\tilde{u}_{n}-\tilde{v}_{n}\right) d x .
\end{aligned}
$$

From the definition of the Dirichlet-to-Neumann map, this generates

$$
\begin{aligned}
& \int_{\Omega \backslash \bar{D}}\left|\nabla w_{n}\right|^{2} d x=\left\langle(\overbrace{\left.\Lambda_{\partial D, \sigma}-\Lambda_{0,0}\right) f_{n}}, f_{n}\rangle-\int_{\partial D} u_{n} \frac{\partial\left(\tilde{u}_{n}-\tilde{v}_{n}\right)}{\partial \nu} d s\right. \\
& +\int_{\Omega \backslash \bar{D}} k^{2} u_{n}\left(\tilde{u}_{n}-\tilde{v}_{n}\right) d x-\int_{\Omega \backslash \bar{D}} \nabla v_{n} . \nabla \tilde{u}_{n} d x+\int_{\Omega \backslash \bar{D}}\left|\nabla v_{n}\right|^{2} d x .
\end{aligned}
$$

Applying $\nabla v_{n} \cdot \nabla \tilde{u}_{n}=\nabla \cdot\left(\tilde{u}_{n} \nabla v_{n}\right)-\tilde{u}_{n} \Delta v_{n}$ in this expression, we get

$$
\begin{aligned}
& -\int_{\Omega \backslash \bar{D}} \nabla v_{n} \cdot \nabla \tilde{u}_{n} d x+\int_{\Omega \backslash \bar{D}}\left|\nabla v_{n}\right|^{2} d x=-\int_{\partial \Omega} \tilde{u}_{n} \frac{\partial v_{n}}{\partial \nu} d s+\int_{\partial D} \tilde{u}_{n} \frac{\partial v_{n}}{\partial \nu} d s \\
& -k^{2} \int_{\Omega \backslash \bar{D}} \tilde{u}_{n} v_{n} d x+\int_{\Omega}\left|\nabla v_{n}\right|^{2} d x-\int_{D}\left|\nabla v_{n}\right|^{2} d x .
\end{aligned}
$$

Also by applying $\left|\nabla v_{n}\right|^{2}=\nabla \cdot\left(\tilde{v}_{n} \nabla v_{n}\right)-\tilde{v}_{n} \Delta v_{n}$ and noticing $w_{n}=u_{n}-v_{n}=0$ on $\partial \Omega$ in (6.9), we get

$$
\begin{aligned}
-\int_{\Omega \backslash \bar{D}} \nabla v_{n} \cdot \nabla \tilde{u}_{n} d x+\int_{\Omega \backslash \bar{D}}\left|\nabla v_{n}\right|^{2} d x= & \int_{\partial D} \tilde{u}_{n} \frac{\partial v_{n}}{\partial \nu} d s-k^{2} \int_{\Omega \backslash \bar{D}} \tilde{u}_{n} v_{n} d x \\
& -\int_{\Omega} \tilde{v}_{n} \Delta v_{n} d x-\int_{D}\left|\nabla v_{n}\right|^{2} d x
\end{aligned}
$$

Now inserting (6.10) into (6.8) yields

$$
\begin{aligned}
& \int_{\Omega \backslash \bar{D}}\left|\nabla w_{n}\right|^{2} d x \\
= & \left\langle\left(\Lambda_{\partial D, \sigma}-\Lambda_{0,0}\right) \tilde{f}_{n}, f_{n}\right\rangle+\int_{\partial D}\left(\tilde{u}_{n} \frac{\partial v_{n}}{\partial \nu}-u_{n} \frac{\partial\left(\tilde{u}_{n}-\tilde{v}_{n}\right)}{\partial \nu}\right) d s+k^{2} \int_{D}\left|v_{n}\right|^{2} d x \\
& +k^{2} \int_{\Omega \backslash D}\left[u_{n}\left(\tilde{u}_{n}-\tilde{v}_{n}\right)-\tilde{u}_{n} v_{n}+v_{n} \tilde{v}_{n}\right] d x-\int_{D}\left|\nabla v_{n}\right|^{2} d x
\end{aligned}
$$


that is,

$$
\begin{aligned}
& \langle\overbrace{\left(\Lambda_{\partial D, \sigma}-\bar{\Lambda}_{0,0}\right) f_{n}}, f_{n}\rangle \\
= & \int_{\Omega \backslash \bar{D}}\left(\left|\nabla w_{n}\right|^{2}-k^{2}\left|w_{n}\right|^{2}\right) d x+\int_{D}\left(\left|\nabla v_{n}\right|^{2}-k^{2}\left|v_{n}\right|^{2}\right) d x \\
& -\int_{\partial D}\left(\tilde{u}_{n} \frac{\partial v_{n}}{\partial \nu}-u_{n} \frac{\partial\left(\tilde{u}_{n}-\tilde{v}_{n}\right)}{\partial \nu}\right) d s \\
= & \int_{\Omega \backslash \bar{D}}\left(\left|\nabla w_{n}\right|^{2}-k^{2}\left|w_{n}\right|^{2}\right) d x+\int_{D}\left(\left|\nabla v_{n}\right|^{2}-k^{2}\left|v_{n}\right|^{2}\right) d x \\
& -\int_{\partial D_{2}}\left(\tilde{u}_{n} \frac{\partial v_{n}}{\partial \nu}+u_{n} \frac{\partial \tilde{v}_{n}}{\partial \nu}\right) d s-\int_{\partial D_{3}}\left(\tilde{u}_{n} \frac{\partial v_{n}}{\partial \nu}+u_{n} \frac{\partial \tilde{v}_{n}}{\partial \nu}-i \sigma\left|u_{n}\right|^{2}\right) d s .
\end{aligned}
$$

6.2. Estimate for $w$. Here we prove the fourth estimate in Theorem 5.2 .

For a given needle $c \in \Omega \backslash \bar{D}$, put $x_{0}=c(t) \in \Omega \backslash \bar{D}$ and let $a \in \partial D$ be the point at which the needle $c$ first hits $\partial D$. Since other cases can be handled analogously, we only consider the case $a \in \partial D_{3}$. Suppose $x_{0}$ is very close to $a$. Consider two families of functions $\left\{w\left(\cdot, x_{0}\right)\right\},\left\{z\left(\cdot, x_{0}\right)\right\}$ depending on $x_{0}$ in some function space $X$. We denote by $w\left(\cdot, x_{0}\right) \sim z\left(\cdot, x_{0}\right)$ in $X$ if $\left\{w\left(\cdot, x_{0}\right)-z\left(\cdot, x_{0}\right)\right\}$ is a bounded set in $X$ for $x_{0}$ very close to $a$.

Let $G_{0}\left(x-x_{0}\right)=\frac{1}{4 \pi\left|x-x_{0}\right|}$. Then it is easy to see that

$$
\left(\partial_{\nu}+i \sigma\right) G\left(x-x_{0}\right) \sim\left(\partial_{\nu}+i \sigma\right) G_{0}\left(x-x_{0}\right)
$$

in $L^{2}(\partial D)$; hence

$$
w\left(\cdot, x_{0}\right) \sim w_{0}\left(\cdot, x_{0}\right) \quad \text { in } H^{1}(\Omega \backslash \bar{D}),
$$

where $w=w\left(\cdot, x_{0}\right) \in H^{1}(\Omega \backslash \bar{D})$ is the solution to (3.6) and $w_{0}=w_{0}\left(\cdot, x_{0}\right) \in H^{1}(\Omega \backslash \bar{D})$ is the solution to

$$
\begin{cases}\Delta w_{0}+k^{2} w_{0}=0, & \text { in } \Omega \backslash \bar{D}, \\ \left(\frac{\partial}{\partial \nu}+i \sigma\right) w_{0}=-\left(\frac{\partial}{\partial \nu}+i \sigma\right) G_{0}\left(\cdot-x_{0}\right), & \text { on } \partial D_{3}, \\ w_{0}=0, & \text { on } \partial \Omega .\end{cases}
$$

Here, we did not write the other boundary conditions on $\partial D_{j}(j=1,2)$. Since $x_{0}$ is close to $a \in \partial D_{3}$, the other boundary conditions will not affect the behavior of $w\left(\cdot, x_{0}\right)$ as $x_{0} \rightarrow a$. So, hereafter we will suppress the other boundary conditions.

By the Sobolev embedding $H^{1 / 2}(\partial D) \hookrightarrow L^{r}(\partial D)$ with $2 \leq r \leq 4$ and the Hölder inequality, for any $q\left(\frac{4}{3} \leq q \leq 2\right)$, there exists a constant $C>0$ such that

$$
\begin{aligned}
\left|\int_{\partial D} i \sigma(x) G_{0}\left(x-x_{0}\right) \phi d s\right| & \leq\left\|i \sigma G_{0}\left(\cdot-x_{0}\right)\right\|_{L^{q}(\partial D)}\|\phi\|_{L^{r}(\partial D)} \\
& \leq C\left\|i \sigma G_{0}\left(\cdot-x_{0}\right)\right\|_{L^{q}(\partial D)}\|\phi\|_{H^{1 / 2}(\partial D)}
\end{aligned}
$$

for $\phi \in H^{1 / 2}(\partial D)$, where $\frac{1}{r}=1-\frac{1}{q}$ with $\frac{4}{3} \leq q<2$.

Hence $i \sigma G_{0}\left(\cdot-x_{0}\right) \sim 0$ in $H^{-1 / 2}(\partial D)$, and by the well-posedness of our boundary value problem, this implies

$$
w_{0}\left(\cdot, x_{0}\right) \sim w_{1}\left(\cdot, x_{0}\right) \quad \text { in } H^{1}(\Omega \backslash \bar{D}),
$$




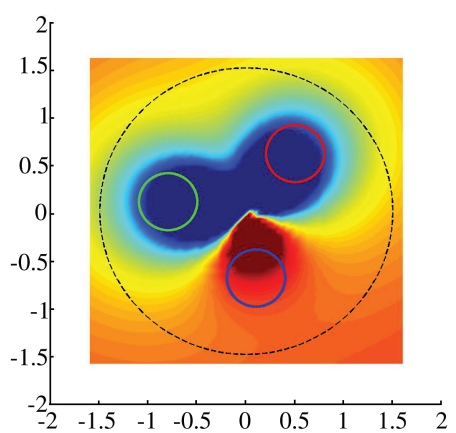

(a) $\Re I\left(f_{n, z}\right)$
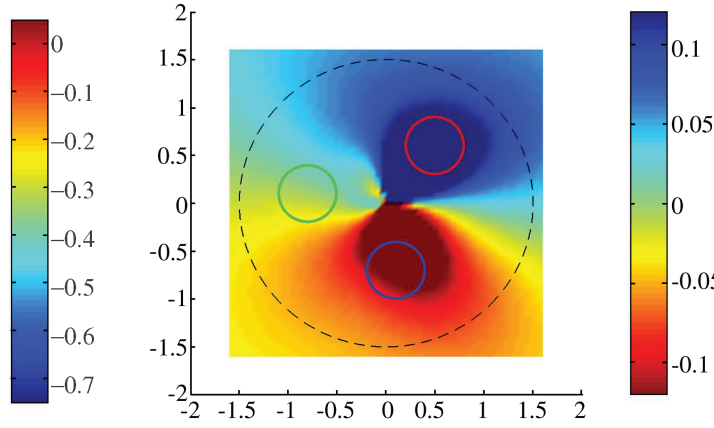

(b) $\Im I\left(f_{n, z}\right)$

FIG. 1. Results for the case of three different types of obstacles. (a): the real part of the indicator function cut by $C_{1}=-0.75$ and $C_{2}=0.05$; (b): the imaginary part of the indicator function cut by $C_{3}=-0.12$ and $C_{4}=0.12$. We take $k=1.0$ and $\alpha=1 e-8$ in the computations.

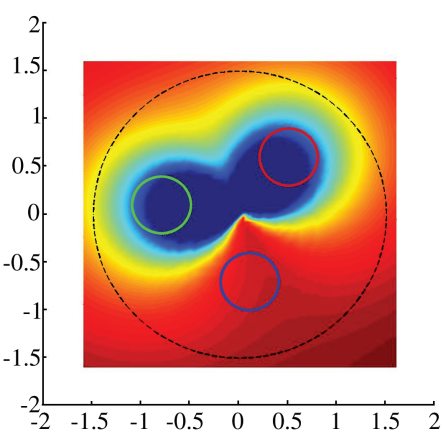

(a) $\Re I\left(f_{n, z}\right)$
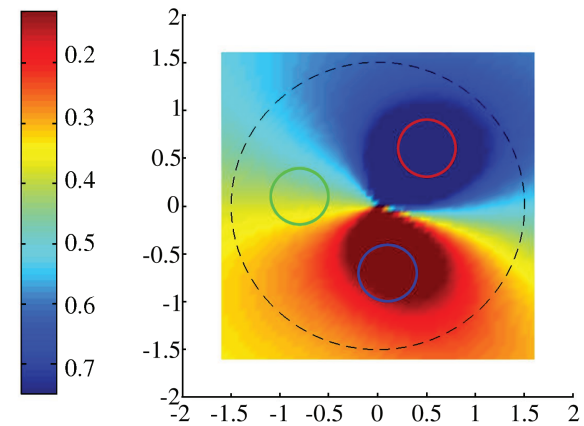

(b) $\Im I\left(f_{n, z}\right)$

Fig. 2. Results for the three-obstacles case with the maximum $10 \%$ random error in the Dirichlet-to-Neumann map. (a): the real part; (b): the imaginary part. In this computation, we take $k=1.0$ and $\alpha=1 e-4$. 
where $w_{1}=w_{1}\left(\cdot, x_{0}\right) \in H^{1}(\Omega \backslash \bar{D})$ is the solution to

$$
\begin{cases}\Delta w_{1}+k^{2} w_{1}=0, & \text { in } \Omega \backslash \bar{D}, \\ \left(\frac{\partial}{\partial \nu}+i \sigma(x)\right) w_{1}=-\frac{\partial}{\partial \nu} G_{0}\left(\cdot-x_{0}\right), & \text { on } \partial D_{3}, \\ w_{1}=0, & \text { on } \partial \Omega .\end{cases}
$$

Now consider the solution $w_{2}=w_{2}\left(\cdot, x_{0}\right) \in H^{1}(\Omega \backslash \bar{D})$ to

$$
\begin{cases}\Delta w_{2}=0, & \text { in } \Omega \backslash \bar{D}, \\ \frac{\partial}{\partial \nu} w_{2}=-\frac{\partial}{\partial \nu} G_{0}\left(\cdot-x_{0}\right), & \text { on } \partial D_{3}, \\ w_{2}=0, & \text { on } \partial \Omega .\end{cases}
$$

For this problem, we have

Claim 1: $i \sigma(x) w_{2}\left(\cdot, x_{0}\right) \sim 0$ in $H^{-1 / 2}(\partial D), \quad w_{2}\left(x, x_{0}\right) \sim 0$ in $L^{2}(\Omega \backslash \bar{D})$.

Proof. The proof given here also gives a more precise estimate for $w_{2}$, which will be used in the sequel.

Let $y=\left(y_{1}, y_{2}, y_{3}\right)=\left(y_{1}\left(x, x_{0}\right), y_{2}\left(x, x_{0}\right), y_{3}\left(x, x_{0}\right)\right)$ be boundary normal coordinates near point $a$ such that

$$
y(a)=0, \quad J(x):=\frac{\partial\left(y\left(x, x_{0}\right)\right)}{\partial x}=I(\text { identity matrix })
$$

at $x=x_{0}$ and $D=\left\{y_{1}<0\right\}$ locally near point $a$. Also, let $y\left(x_{0}\right)=y_{0}$,

$$
\begin{aligned}
& A(x):=|\operatorname{det} J(x)|^{-1} J(x)(J(x))^{T}, \quad x\left(y\left(x, x_{0}\right) ; x_{0}\right)=x, \\
& \underline{A}(y):=A\left(x\left(y ; x_{0}\right)\right), \quad \underline{u}(y):=u\left(x\left(y ; x_{0}\right)\right) .
\end{aligned}
$$

Then it is easy to see that

(1) $\underline{A}(y) \in C^{1}$ near $y=0$;

(2) $\Delta u=0$ near point $a \Longleftrightarrow \nabla \cdot \underline{A} \nabla \cdot \underline{u}=0$ near 0 ;

(3) $\delta\left(x\left(y ; x_{0}\right)-x_{0}\right)=\delta\left(y-y_{0}\right)$;

(4) $\partial_{\nu}=\partial_{y_{1}}$.

In order to simplify the description of our argument, from now on we extend $x\left(y ; x_{0}\right)$ and $\underline{A}(y)$ to an open ball $V \subset R^{3}$ centered at $y=0$ without destroying their regularities and the positivity of $\underline{A}(y)$. By a direct estimate, we can easily see that

$$
\underline{G}_{0}\left(y ; y_{0}\right) \sim G_{0}\left(y-y_{0}\right) \quad \text { in } H^{1}(V),
$$

where we have adopted the convention $y_{0}=y\left(x_{0} ; x_{0}\right)$.

Now consider the solution $\underline{w}_{2}^{0} \in H^{1}\left(V \cap R_{+}^{3}\right)$ to

$$
\begin{cases}\Delta \underline{w}_{2}^{0}=0, & \text { in } y_{1}>0, \\ \partial_{y_{1}} \underline{w}_{2}^{0}=-\partial_{y_{1}} G_{0}\left(y-y_{0}\right), & \text { on } y_{1}=0, \\ \mathrm{DC} & \end{cases}
$$

and put $\underline{w}_{2}(y):=w_{2}\left(x\left(y, x_{0}\right)\right)$, where DC means that we put the Dirichlet condition on the rest of the part of the boundary. If we can prove

Claim 2: $\nabla \cdot\left(\underline{A}(y)-\underline{A}\left(y_{0}\right)\right) \nabla \underline{w}_{2}^{0} \sim 0$ in $W^{*}$, where $W:=\left\{\phi \in H^{1}\left(V \cap R_{+}^{3}\right)\right) \mid w=$ 0 on $\left.\partial V \cap R_{+}^{3}\right\}$ and $W^{*}$ is its dual space.

Then we have

$$
\underline{w}_{2} \sim \underline{w}_{2}^{0} \quad \text { in } H^{1}\left(V \cap R_{+}^{3}\right)
$$


by observing that $z:=\left(\underline{w}_{2}-\underline{w}_{2}^{0}\right)+\left(\underline{G}_{0}-G_{0}\left(\cdot-y_{0}\right)\right)$ satisfies

$$
\begin{cases}\nabla \cdot \underline{A} \nabla z=-\nabla \cdot\left(\underline{A}(y)-\underline{A}\left(y_{0}\right)\right) \nabla\left(\underline{w}_{2}^{0}+G_{0}\left(\cdot-y_{0}\right)\right), & \text { in } V \cap R_{+}^{3}, \\ \partial_{y_{1}} z=0, & \text { on } y_{1}=0 .\end{cases}
$$

The proof for Claim 2 will be given in Section 6.3. Therein we also give a precise expression for $\underline{w}_{2}^{0}(y)$. Then, this expression and (6.20) imply Claim 1 . The proof is complete.

Now we can see that

$$
w_{1}\left(\cdot, x_{0}\right) \sim w_{2}\left(\cdot, x_{0}\right) \quad \text { in } H^{1}(\Omega \backslash \bar{D})
$$

from Claim 1 and the well-posedness of our boundary value problem.

Now summing up (6.13), (6.16), (6.20) and (6.22), as well as the expression of $\widetilde{w}_{2}^{0}(y)$ given in the next subsection, we have

$$
\int_{\partial D}\left|w\left(x, x_{0}\right)\right|^{2} d s \leq C\left(\int_{\partial D}|G(x-c(t))|^{2} d s+1\right),
$$

which completes the proof of the fourth estimate in Theorem 5.2 .

6.3. Proof for Claim 2.

Proof. Let $y_{0}=\left(y_{01}, y_{02}, y_{03}\right)=\left(y_{01}, y_{0}^{\prime}\right)$. Then it is well known that $H(y)=$ $H\left(y ; y_{0}\right)=G_{0}\left(y-y_{0}\right)$ can be given by

$$
H(y)= \begin{cases}H_{+}(y)=H_{+}\left(y ; y_{0}\right), & \text { in } y_{1}>y_{01}, \\ H_{-}(y)=H_{-}\left(y ; y_{0}\right), & \text { in } y_{1}<y_{01},\end{cases}
$$

with the solution $H_{ \pm}(y)$ to

$$
\left\{\begin{array}{l}
\Delta H_{ \pm}(y)=0, \quad \text { in } \pm\left(y_{1}-y_{01}\right)>0, \\
\left.H_{+}(y)\right|_{y_{1}=y_{01}+0}=\left.H_{-}(y)\right|_{y_{1}=y_{01}-0}, \\
\left.\partial_{y_{1}} H_{+}(y)\right|_{y_{1}=y_{01}+0}-\left.\partial_{y_{1}} H_{-}(y)\right|_{y_{1}=y_{01}-0}=-\delta\left(y^{\prime}-y_{0}^{\prime}\right) .
\end{array}\right.
$$

Denote by $\Gamma_{ \pm}\left(y_{1}, \eta^{\prime}\right)$ and $w\left(y_{1}, \eta^{\prime}\right)$ the Fourier transforms of $\hat{H}_{ \pm}(y)$ and $\widetilde{w}_{2}^{0}(y)$ with respect to $y^{\prime}$, respectively. Then, $\Gamma_{ \pm}^{\prime}:=e^{i y_{0}^{\prime} \cdot \eta^{\prime}} \Gamma_{ \pm}$and $w^{\prime}:=e^{i y_{0}^{\prime} \cdot \eta^{\prime}} w$ satisfy

$$
\left\{\begin{array}{l}
\left(\partial_{y_{1}}^{2}-\left|\eta^{\prime}\right|^{2}\right) \Gamma_{ \pm}^{\prime}=0, \quad \text { in } \pm\left(y_{1}-y_{01}\right)>0 \\
\left.\Gamma_{+}^{\prime}\right|_{y_{1}}=y_{01}+0=\left.\Gamma_{-}^{\prime}\right|_{y_{1}=y_{01}-0} \\
\left.\partial_{y_{1}} \Gamma_{+}^{\prime}\right|_{y_{1}=y_{01}+0}-\left.\partial_{y_{1}} \Gamma_{-}^{\prime}\right|_{y_{1}=y_{01}-0}=-1
\end{array}\right.
$$

and

$$
\begin{cases}\left(\partial_{y_{1}}^{2}-\left|\eta^{\prime}\right|^{2}\right) w^{\prime}=0, & \text { in } y_{1}>0 \\ \partial_{y_{1}} w^{\prime}=-\partial_{y_{1}} \Gamma_{-}^{\prime}, & \text { on } y_{1}=0\end{cases}
$$

respectively. $\Gamma_{ \pm}^{\prime}=\Gamma_{ \pm}^{\prime}\left(y_{1}\right)$ is given by

$$
\Gamma_{ \pm}^{\prime}\left(y_{1}\right)=2^{-1}\left|\eta^{\prime}\right|^{-1} e^{\mp\left(y_{1}-y_{01}\right)\left|\eta^{\prime}\right|} .
$$

Hence $w^{\prime}=w^{\prime}\left(y_{1}\right)=2^{-1}\left|\eta^{\prime}\right|^{-1} e^{-\left(y_{1}+y_{01}\right)\left|\eta^{\prime}\right|}$. Comparing these two formulas, we have

$$
\widetilde{w}_{2}^{0}(y)=H_{+}\left(y_{1}, y^{\prime} ;-y_{01}, y_{0}^{\prime}\right)=\frac{1}{4 \pi \sqrt{\left(y_{1}+y_{01}\right)^{2}+\left|y^{\prime}-y_{0}^{\prime}\right|^{2}}} .
$$

This completes the proof of Claim 2 . 
7. Numerical performance. For simplicity, we present the realizations of the probe method for multiple obstacles in $\mathbb{R}^{2}$. The numerical realization of the probe method was first given in 8 for one sound-soft obstacle and later given in 2] for one obstacle with impedance boundary.

In this section, we give the numerical performance of the probe method for multiple obstacles for which the theoretical analysis is given in this paper. We present the scheme for the reconstructions of multiple obstacles based on the scheme given in [8]. Although the theoretical analysis only gives the behavior of the indicator function $I(t, c)$ given by (3.3) when $c(t)$ approaches the boundaries of the obstacles from the outside, we compute $I_{n}(t, c)$ for fixed $n \gg 1$ and all $c(t)$.

Since our purpose is to show the efficiency of the probe method, in this section, we generate the Neumann data by simulation directly, rather than from the far-field pattern. That is, we obtain the solution to (2.2) by assuming that the obstacle $D$ is known. Then we can solve this problem by potential theory to generate the Neumann data on $\partial \Omega$. The details for this procedure in the one-obstacle case may be found in [2, 8, 21].

The essential part of the numerical performance of the probe method for multiple obstacles is the Runge approximation. For this, we use the scheme proposed in [8] which is as follows. To start with, we consider the minimum norm solution of the equation

$$
H g_{z}(x):=\int_{\mathbb{S}} e^{i k x \cdot d} g_{z}(d) d s(d)=G(\cdot, z)
$$

for a given $z \in \Omega$. Theoretically, the approximation domain is

$$
E(z)=E(c(t)):=\Omega \backslash\{c(\tau): 0<\tau \leq t\} .
$$

However, this approximation domain $E(z)$ is unstable in numerics due to the approximation of the singular function $G(\cdot, z)$ in $E(z)$ with strong non-convexity as $z \rightarrow \partial D$. So we choose relatively simple domains in numerical implementations.

More precisely, we consider approximation domains with fixed shape in the form

$$
E(z)=\left\{M(z) x+z: x \in E_{0}, M(z) \in S O(2, \mathbb{R})\right\}
$$

with a fixed approximation domain $E_{0}:=E(0)$ corresponding to $z=0$, where we assumed $0 \in \Omega$ and $S O(2, \mathbb{R})$ represents the set of $2 \times 2$ unitary orthogonal matrices.

Then, for any $z \in \Omega$ with corresponding approximation domain $E(z)$ of the form (7.3), the density

$$
g_{\alpha, z}=e^{-i \kappa z \cdot d} g_{\alpha, 0}\left(M(z)^{t} d\right)
$$

satisfies a Herglotz wave function such that

$$
\left\|H g_{\alpha, z}-G(\cdot, z)\right\|_{L^{2}(\partial E(z))}<\delta .
$$

This property reduces the complexity of calculating the densities $g_{\alpha, z}$ by choosing a fixed base domain $E_{0}$, from which all approximation domains $E(z)$ are generated by a rotation and a translation.

The procedure of realization of the probe method is as follows:

(1) Choose a test domain $E_{0}$ such that $0 \notin E_{0}$ large enough and calculate $g_{\alpha, 0}$ for a proper choice of $\alpha$, and then compute $g_{\alpha, z_{j}}$ via (7.4) for evaluation points $z_{j}=c\left(t_{j}\right) \in \Omega$ with $j=1, \ldots, N$. 
(2) Fix $n \gg 1$ and calculate $f_{n, j}:=\left.v_{n, z_{j}}\right|_{\partial \Omega}$ for $j=1, \ldots, N$ with $v_{n, z_{j}}=H g_{\alpha, z_{j}}$, where $\frac{1}{n}$ is the discrepancy of the minimum norm solution, and $\alpha$ is chosen by trial and error.

(3) Solve the direct obstacle boundary value problem and the direct obstacle-free boundary value problem with $f=f_{n, j}$ to simulate $\Lambda_{D} f_{n, j}, \Lambda_{0} f_{n, j}$ for $j=$ $1, \ldots, N$.

(4) Calculate the approximation $I\left(f_{n, j}\right) \approx \hat{I}_{n}\left(z_{j}\right)$ with $c(t)=z_{j}$ given by (3.3) for $j=1, \ldots, N$.

(5) Observe the behavior of the indicator function as $c(t)$ moves along a needle.

Example: Three-obstacle case with different type of boundary.

We choose $\Omega$ as a circular domain with radius $r_{\Omega}=1.5$ and the three obstacles are

$$
\begin{aligned}
& \partial D_{1}:=\{(x, y):(x, y)=(0.1+0.3 \cos t,-0.7+0.3 \sin t) ; t \in[0,2 \pi)\}, \\
& \partial D_{2}:=\{(x, y):(x, y)=(-0.8+0.3 \cos t, 0.1+0.3 \sin t) ; t \in[0,2 \pi)\}, \\
& \partial D_{3}:=\{(x, y):(x, y)=(0.5+0.3 \cos t, 0.6+0.3 \sin t) ; t \in[0,2 \pi)\}
\end{aligned}
$$

with sound-soft, sound-hard and impedance boundary respectively.

For the sake of computational simplicity, we choose a square $S=(-1.6,1.6) \times$ $(-1.6,1.6)$ which includes $\Omega$, and divide $S$ into $60 \times 60$ non-overlapping elements. Then $\left\{z_{j}\right\}_{j=1}^{3600}$ is the set for the centers of all elements. Furthermore, we take the impedance coefficient $\sigma=1.0$ for the obstacle $D_{3}$.

Figure 1(a) shows the real part of the indicator function cut by the constants $C_{1}=$ -0.75 and $C_{2}=0.05$. Figure $1(\mathrm{~b})$ shows the imaginary part cut by the constants $C_{3}=-0.12$ and $C_{4}=0.12$. Here, for instance, $C_{1}$ and $C_{2}$ are the cutting values of the lower and upper bounds of the level sets of the real part of the indicator function, respectively. Hence, Figure 1(a) shows the level sets of the real part of the indicator function between the lower level $C_{1}$ and the upper level $C_{2}$. The meanings of $C_{3}, C_{4}$ are likewise those of $C_{1}, C_{2}$.

From the real part of the indicator function, the cutoff negative value in Figure 1(a) gives an enclosure of the two obstacles, the sound-hard obstacle and the impedance obstacle. We observe that the boundary of this enclosure is close to some parts of the exact boundaries of the two obstacles, and the cutoff positive value in Figure 1(a) gives a very rough shape of the sound-soft obstacle. From the imaginary part, the cutoff positive value in Figure 1(b) gives a rough shape of the obstacle with impedance boundary and the cutoff negative value in Figure 1(b) gives a rough shape of the sound-soft obstacle.

Note that the red circle represents the exact boundary of the obstacle with impedance boundary, the green circle is the exact boundary of the sound-hard obstacle and the blue circle is the exact boundary of the sound-soft obstacle in Figure 1 and Figure 2,

From Figure 1(a) and Figure 1(b), we easily recognize that there are three different types of obstacles and identify some part of the boundary for the sound-hard obstacle and the obstacle with impedance boundary. Moreover, we obtain the rough shapes for the three obstacles.

Figure 2 shows the numerical results when we add the maximum $10 \%$ random error to the Dirichlet-to-Neumann map. We observe that the sound-soft obstacle cannot be 
identified from the real part of the indicator function, but the imaginary part can give a rough shape for this obstacle. Therefore, we have similar results as in the no error case.

On the other hand, it can be found from these examples that we can reconstruct a little more than a convex hull of multiple obstacles only; the neighboring parts of different obstacles are hard to recover. We guess that the reason is due to the approximation accuracy of the fundamental solution in the approximated domain corresponding to the needle points nearing this neighboring part. Roughly speaking, it is not so easy to approximate the fundamental solution to an arbitrary accuracy as the approximation domain becomes more and more like a cone-like domain (2]). So it can be concluded from the numerical performance here that the probe method can be applied to generate a good initial guess for other iterative inversion schemes. It seems not easy to identify the shapes of the multiple obstacles exactly by this method itself from the numerical point of view.

\section{REFERENCES}

[1] J. Cheng, J.J. Liu, G. Nakamura, Recovery of the shape of an obstacle and the boundary impedance from the far-field pattern, J. Math. Kyoto University, 43(2003), 165-186. MR2028705(2004k:35395)

[2] J. Cheng, J.J. Liu, G. Nakamura, The numerical realization of the probe method for the inverse scattering problems from the near-field data, Inverse Problems, 21(2005), 839-855. MR2144808 (2006b:35335)

[3] D.L. Colton, A. Kirsch, A simple method for solving inverse scattering problems in the resonance region, Inverse Problems, 12(1996), 383-393. MR 1402098(97d:35032)

[4] D.L. Colton, A. Kirsch, The determination of the surface impedance of an obstacle from measurements of the far field pattern, SIAM J. Appl. Math., 41(1981), 8-15. MR622870 (83d:35157)

[5] D.L. Colton, R. Kress, Integral Equation Methods in Scattering Theory, John Wiley \& Sons, Inc., New York (1983). MR700400 (85d:35001)

[6] D.L. Colton, R. Kress, Inverse Acoustic and Electromagnetic Scattering Theory, Springer-Verlag, Berlin (1998). MR.1635980 (99c:35181)

[7] M.A. Epton, B. Dembart, Multipole translation theory for the three-dimensional Laplace and Helmholtz equations, SIAM J. Sci. Comput., 16(1995), 865-897. MR.1335895 (96f:35023)

[8] K. Erhard and R. Potthast, A numerical study of the probe method, SIAM J. Sci. Comput., 28(2006), 1597-1612. MR2272180 (2007h:35349)

[9] N. I. Grinberg, Obstacle visualization via the factorization method for the mixed boundary value problem, Inverse Problems, 18(2002), 1687-1704. MR1955913 (2004c:35313)

[10] M. Ikehata, Reconstruction of an obstacle from the scattering amplitude at a fixed frequency, Inverse Problems, 14(1998), 949-954. MR1642552 (99e:35242)

[11] M. Ikehata, Reconstruction of the shape of the inclusion by boundary measurements, Commun. in PDE, 23(1998), 1459-1474. MR.1642619 (99f:35222)

[12] M. Ikehata, Reconstruction of obstacle from boundary measurements, Wave Motion, 30(1999), 205223. MR.1708203 (2000i:35214)

[13] M. Ikehata, G. Nakamura, Slicing of a three-dimensional object from boundary measurements, Inverse Problems, 15(1999), 1243-1253. MR1715362 (2000j:78004)

[14] M. Ikehata, G. Nakamura, K. Tanuma, Identification of the shape of the inclusion in the anisotropic elastic body, Appl. Anal., 72(1999), 17-26. MR1775432 (2001d:74026)

[15] A. Kirsch, Characterization of the shape of a scattering obstacle using the spectral data of the far field operator, Inverse Problems, 14(1998), 1489-1512. MR1662460 (99k:35193)

[16] A. W. Kedzierawski, The determination of the surface impedance of an obstacle, Proc. Edinburgh Math. Soc., 36(1993), 1-15. MR.1200183 (93m:35181)

[17] N. I. Grinberg and A. Kirsch, The factorization method for obstacles with a priori separated sound-soft and sound-hard parts, Math. Computers in Simulation, 66(2004),267-279. MR2079578 (2005b:35212) 
[18] R. Kress, A. Zinn, On the numerical solution of the three-dimensional inverse obstacle scattering problem, J. Comput. Appl. Math., 42(1992), 49-61. MR.1181580(93f:65052)

[19] R. Kress, On the numerical solution of a hypersingular integral equation in scattering theory, J. Comput. Appl. Math., 61(1995), 345-360. MR1361270 (96h:65166)

[20] J.J. Liu, Inverse scattering for the problem with impedance-type boundary, J. Partial Differential Equations, 13(2000), 279-288. MR.1779075 (2001m:35320)

[21] J.J. Liu, Determination of Dirichlet-to-Neumann map for a mixed boundary problem, Appl. Math. Comput., 161(2005), 843-864. MR2113524

[22] L. Monch, A Newton method for solving the inverse scattering problem for a sound-hard obstacle, Inverse Problems, 12(1996), 309-323. MR1391541 (97e:65058)

[23] R. Potthast, Stability estimates and reconstructions in inverse acoustic scattering using singular sources, J. Comput. Appl. Math., 114(2000), 247-274. MR.1737077(2000k:35297)

[24] R.T. Smith, An inverse acoustic scattering problem for an obstacle with an impedance boundary condition, J. Math. Anal. Appl., 105 (1985), 333-356. MR778470 (86f:35059) 